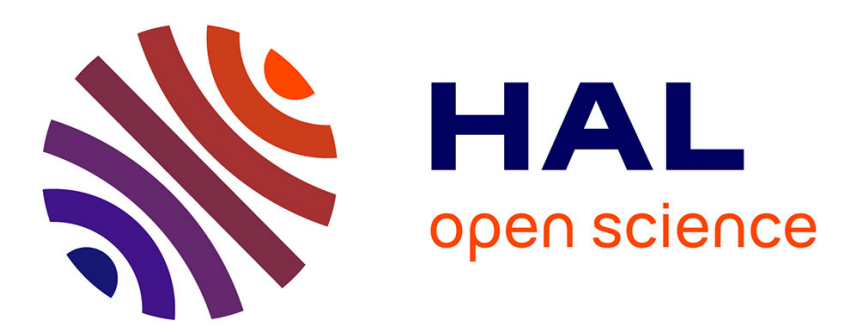

\title{
Directed Homology Theories and Eilenberg-Steenrod Axioms
}

Jérémy Dubut, Eric Goubault, Jean Goubault-Larrecq

\section{To cite this version:}

Jérémy Dubut, Eric Goubault, Jean Goubault-Larrecq. Directed Homology Theories and EilenbergSteenrod Axioms. Applied Categorical Structures, 2017, 25 (5), pp.775-807. 10.1007/s10485-0169438-y . hal-03189639

\section{HAL Id: hal-03189639 \\ https://hal.inria.fr/hal-03189639}

Submitted on 9 Jun 2021

HAL is a multi-disciplinary open access archive for the deposit and dissemination of scientific research documents, whether they are published or not. The documents may come from teaching and research institutions in France or abroad, or from public or private research centers.
L'archive ouverte pluridisciplinaire $\mathbf{H A L}$, est destinée au dépôt et à la diffusion de documents scientifiques de niveau recherche, publiés ou non, émanant des établissements d'enseignement et de recherche français ou étrangers, des laboratoires publics ou privés. 


\title{
Directed homology theories and Eilenberg-Steenrod axioms
}

\author{
Jérémy Dubut ${ }^{1,2}$, Éric Goubault ${ }^{2}$ and Jean Goubault-Larrecq ${ }^{1}$ \\ ${ }^{1}$ LSV, ENS Cachan, CNRS, Université Paris-Saclay, \\ 94235 Cachan, France \\ jdubut@ens-cachan.fr, goubault@lsv.ens-cachan.fr \\ ${ }^{2}$ LIX, Ecole Polytechnique, Palaiseau, France \\ goubault@lix.polytechnique.fr
}

\begin{abstract}
In this paper, we define and study a homology theory, that we call "natural homology", which associates a natural system of abelian groups to every space in a large class of directed spaces and precubical sets. We show that this homology theory enjoys many important properties, as an invariant for directed homotopy. Among its properties, we show that subdivided precubical sets have the same homology type as the original ones ; similarly, the natural homology of a precubical set is of the same type as the natural homology of its geometric realization. By same type we mean equivalent up to some form of bisimulation, that we define using the notion of open map. Last but not least, natural homology, for the class of spaces we consider, exhibits very important properties such as Hurewicz theorems, and most of Eilenberg-Steenrod axioms, in particular the dimension, homotopy, additivity and exactness axioms. This last axiom is studied in a general framework of (generalized) exact sequences.
\end{abstract}

\section{Introduction}

We aim at defining and studying thoroughly a new homology theory, well adapted to directed algebraic topology. Directed algebraic topology [20, 14] is a variant of algebraic topology in which the object of study is directed topological spaces, i.e. topological spaces with a preferred direction ("time"), which cannot be reversed. The concepts were introduced originally in concurrency theory $[7,42]$, but they are pervasive to many areas : categorically speaking, directed algebraic topology is deeply linked to the study of $(\infty, 1)$-categories.

Although the theory has now matured, there is still no satisfactory notion of directed homology. The first attempt in that direction was made by Goubault $[21,22]$. Unfortunately, the abelian character of the homology which he defined made the invariants too weak to be useful for characterizing directed spaces 
modulo directed homotopy. In particular, there is no hope for having Hurewiczlike theorems, linking (directed) homology to (directed) homotopy. There are in particular simple directed spaces which are not dihomotopic, and which have the same (directed) homology, in all further (directed) homology theories defined since [21], among which [19, 10, 31].

In order to tackle these problems, while keeping the computationally tractable character of homology, we need to define some form of non-abelian homology theory, as put forward in recent work by Krishnan [33]. Our approach is based on a form of Baues-Wirshing homology theory [38, 39] : we define a natural system of abelian groups, which accounts for the variation of the homology of the so-called trace spaces [43], the topological space of directed paths modulo reparametrization from one point to another, when the start and end points change. This was suggested first in [43] and studied in [9].

In this paper, we give an in-depth account of the first notions defined in [9] and carry on the study of this "natural homology", with a particular focus on Eilenberg-Steenrod axioms, in particular exactness properties, and also Hurewicz types of theorems. This paves the way for practical (directed) homological calculations of concrete directed spaces. Exactness properties are dealt with following the approach for defining generalized homology theories put forward by Grandis $[16,17]$.

Contents We first recap the central notion of trace space, originally introduced by Martin Raussen [43], and give some simple examples, in Section 2.1. Trace spaces contain the essential (directed) homotopical and homological information, but only when a start and end point are fixed. In Section 2.2, we introduce an algebraic structure, of diagrams, which accounts for the change of start and end points, and collect all trace spaces with varying end points. We then compose these diagrams of spaces with the homotopy, respectively homology, functor, to derive our notion of "natural homotopy" and "natural homology". The name comes from the fact that these actually form natural systems (of abelian groups for natural homology) in the sense of Baues and Wirshing $[38,39]$.

In Section 2.3 we recap the notion of precubical set, and of the classical geometric realization of precubical sets (in topological spaces). In order to have a geometric realization which maps onto partially ordered spaces, which are the only types of directed spaces we have been considering in Section 2, we restrict ourselves to particular "geometric" complexes, see Section 2.3. We can then define a notion of natural homology, on these "discrete" spaces, in Section 2.4.

Still, geometric realization does not quite map isomorphically discrete (or combinatorial) natural homology onto the continuous one, at least, not on the nose. In order to relate them, we have to use a notion of bisimulation, which comes originally from concurrency theory $[41,37]$. We begin by recapping the notion of bisimilarity via open maps in Section 3.1, and define a notion of open map for natural homology. We give other characterizations of bisimilarity in Section 3.2, and show in particular that this is equivalent with the notion 
of bisimulation of Definition 3.4, which is simpler to apprehend. We prove in Section 3.3 that indeed, with this notion of bisimilarity, combinatorial and topological homology theories are bisimilar.

Bisimilarity also relates our natural homology, which is a Baues-Wirsing homology theory, with a simpler Hochschild-Mitchell homology theory. This interesting result, both for theoretical and computational purposes, is proved in Section 3.4.

The last part of the paper is dedicated to proving that most of the properties of classical homology theories, such as Hurewicz theorems, or EilenbergSteenrod axioms, which are so convenient for practical calculations in many cases, hold, see Section 4. In this section, we have to use a refined notion of exactness, see Section 4.2.2, following the theory of Grandis [16]. We show that our natural homology theory is homological but not modular in general, which is the main difference between our notion of directed homology with standard homology theories. Still, the (relative homology) exactness axiom of EilenbergSteenrod is valid, as shown in Section 4.2.5. The dimension, homotopy and additivity axioms are simpler to prove, this is done in Section 4.2.1.

We conclude by sketching some possible future work.

\section{Homology of pospaces}

Our aim is to define a notion of homology of so-called directed topological spaces, and more precisely, for the purpose of this paper, of partially-ordered topological spaces, or pospaces [40] (see Definition 2.1). The main ingredient of our homological theories will be the notion of the trace space of a pospace, between two points [43] i.e. the topological space of directed paths modulo reparametrization, see Definition 2.4.

\section{$2.1 \quad$ Trace spaces}

In full generality, directed topology is about d-spaces [20], (pre-)cubical sets [11] or streams [32]. Most of the homological theories we are going to construct can be generalized to such spaces, but we will only prove important properties about these homological theories in some interesting particular cases. In the topological setting, we will restrict ourselves to partially-ordered spaces, whereas in the combinatorial setting, we will restrict our study to particular pre-cubical sets. We indicate in all constructions and theorems whether these are only valid in these particular cases or if this can be generalized.

Partially-ordered spaces, or pospaces, are topological spaces with a simple notion of flow of time :

Definition 2.1 ([40]) Let $\leq$ be a partial order (reflexive, transitive, antisymmetric relation) on $X$, a topological space. We say that $(X, \leq)$ is a pospace if $\leq$ is a closed subspace of $X \times X$. We note PoTop the category of pospace and monotone continuous functions. 
Of course, as for ordinary topological spaces, we are interested in determining whether two pospaces are isomorphic, i.e. when there exists an homeomorphism $f: X \rightarrow Y$, such that $f$ and $f^{-1}$ are non-decreasing maps. This is the classification problem of pospaces. And as for ordinary topological spaces, this is a very hard problem.

We are looking for algebraic invariants of pospaces modulo directed homeomorphisms, in the form of directed homotopical and homological structures. For defining these structures, we need first to construct paths, and spaces of such. In pospaces, we have the classical notion of (continuous) path, but also the new notion of directed path, i.e. a path which agrees with the flow of time, in a pospace :

Definition 2.2 Let $X$ be a topological space, $a, b \in X$. A path from $a$ to $b$ is a continuous function $f:[0,1] \longrightarrow X$ such that $f(0)=a$ and $f(1)=b$. If moreover $X$ is a pospace, $a$ dipath from $a$ to $b$ is a path from $a$ to $b$ which is non-decreasing (when $[0,1]$ is equipped with the usual order).

Let $f$ be $a$ (di)path from $a$ to $b$ and $g$ a (di)path from $b$ to $c$. We define the concatenation $f \star g:[0,1] \longrightarrow X$ by : for all $t \in[0,1 / 2],(f \star g)(t)=f(2 t)$ and for all $t \in[1 / 2,1],(f \star g)(t)=g(2 t-1)$. It is a (di)path from a to $c$.

As usual in algebraic topology, paths are a bit crude - for instance, when it comes to composing them and getting an interesting algebraic structure, i.e. at least associativity of composition, it is necessary to consider them up to reparametrization, at the very least $^{1}$ :

Definition 2.3 ([43]) A non-decreasing reparametrization is a non-decreasing continuous surjection from $[0,1]$ to $[0,1]$.

Let $X$ be a pospace and $p$ and $q$ two dipaths from a to $b$ in $X$. We say that $p$ is reparametrized in $q$ if there exists a non-decreasing reparametrization $\gamma$ such that $p \circ \gamma=q$. The trace of $p$, written $\langle p\rangle$ is the equivalence class modulo non-decreasing reparametrization.

Now we can put together all dipaths from point $a$ to point $b$, modulo nondecreasing reparametrization in a topological space :

Definition 2.4 ([43]) Let $X$ be a pospace and $a$ and $b \in X$. We topologize the set of traces of dipaths from a to $b$, with the compact-open topology. Its quotient $\overrightarrow{\mathfrak{T}}(X)(a, b)$ by reparametrization, with the quotient topology is called the trace space in $X$ from $a$ to $b$.

Trace spaces have good theoretical properties (see for instance [43]) but also computational ones. For instance, it is possible to compute a finite representation of the trace space of the hypercube $I^{n}$ in $\mathbb{R}^{n}$ minus a set of isothetic hypercubes [44].

This construction can be further generalized to non-looping pre-cubical sets, see e.g. [45], with applications to static analysis of concurrent programs [13].

\footnotetext{
${ }^{1}$ Or to consider another class of paths, such as Moore paths.
} 

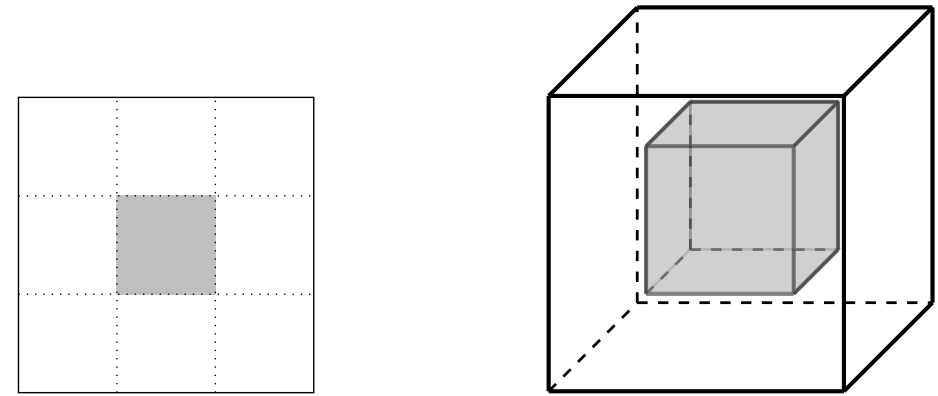

Figure 1: Two simple partially-ordered subspaces of $\mathbb{R}^{2}$ and $\mathbb{R}^{3}$ with componentwise ordering which have non-homotopic trace spaces.

Example 2.1 Let us start with simple examples of trace spaces, pictured in Figure 1 : these are subspaces $X_{1}=[0,1] \times[0,1] \backslash\left[\frac{1}{3}, \frac{2}{3}\right] \times\left[\frac{1}{3}, \frac{2}{3}\right]$ of $\mathbb{R}^{2}$, respectively $X_{2}=[0,1] \times[0,1] \times[0,1] \backslash\left[\frac{1}{3}, \frac{2}{3}\right] \times\left[\frac{1}{3}, \frac{2}{3}\right] \times\left[\frac{1}{3}, \frac{2}{3}\right]$ of $\mathbb{R}^{3}$, with componentwise ordering, i.e. $(x, y) \leq\left(x^{\prime}, y^{\prime}\right)$, respectively $(x, y, z) \leq\left(x^{\prime}, y^{\prime}, z^{\prime}\right)$ if $x \leq x^{\prime}$ and $y \leq y^{\prime}$ with the standard ordering in $\mathbb{R}$, respectively if $x \leq x^{\prime}, y \leq y^{\prime}$ and $z \leq z^{\prime}$ in $\mathbb{R}$.

As shown in [43], $\overrightarrow{\mathfrak{T}}\left(X_{1}\right)((0,0),(1,1))$ is a topological space with two connected components, one is composed of the traces which have dihomotopy type of the path going left and up the hole, the other component is composed of the traces which have the dihomotopy type of the path going right and down the hole. Moreover, the two connected components of $\overrightarrow{\mathfrak{T}}\left(X_{1}\right)((0,0),(1,1))$ are contractible ; it is thus homotopy equivalent to two points.

As shown again in [43], $\overrightarrow{\mathfrak{T}}\left(X_{2}\right)((0,0,0),(1,1,1))$ is homotopy equivalent to the circle $S^{1}$ : there is a unique path up to dihomotopy, hence the trace space $\overrightarrow{\mathfrak{T}}\left(X_{2}\right)((0,0,0),(1,1,1))$ is connected, but there is a finer structure of dihomotopies which accounts for the non simple-connectness character of $\overrightarrow{\mathfrak{T}}\left(X_{2}\right)((0,0$, $0),(1,1,1))$.

\subsection{Homological and homotopical diagrams}

The study of the shape of such spaces of dipaths, when moving $a$ and $b$, will be the essential ingredient of invariants under directed homeomorphisms.

Example 2.2 In Figure 2, we have depicted two spaces on the left, which are built as the gluing of squares (the white ones), each of which is equipped with the product ordering of $\mathbb{R}^{2}$. They will come as the geometric realization of some precubical sets, see Section 2.3. They are not dihomeomorphic spaces since they are already non homotopy equivalent : the fundamental group of the leftmost one, that we call $X$, is the free abelian group on three generators, whereas the fundamental group of the rightmost one, that we call $y$, is the free abelian group on four generators. Consider now our construction of trace space, for $X$ and 

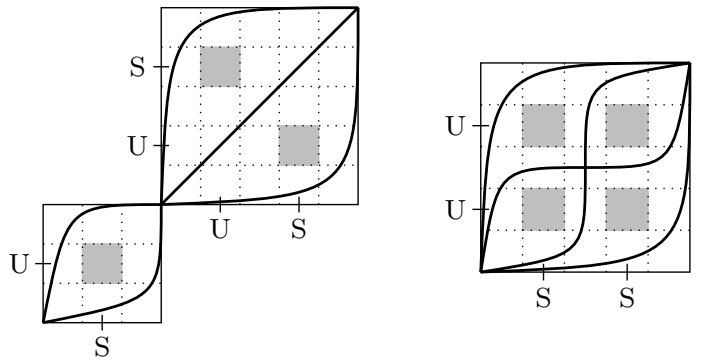

Figure 2: Examples of pospaces coming from non-equivalent concurrent programs.

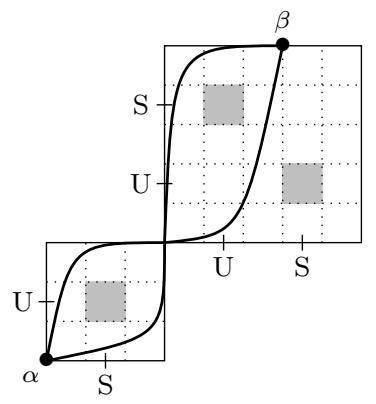

Figure 3: Changing the base points for exhibiting a particular trace space.

$Y$, from the lowest point of $X$ (resp. $Y$ ), that we call $\alpha_{X}$ (resp. $\alpha_{Y}$ ), to the highest point of $X$ (resp. $Y$ ), that we call $\beta_{X}$ (resp. $\alpha_{Y}$ ). We can see easily that $\overrightarrow{\mathfrak{T}}(X)\left(\alpha_{X}, \beta_{X}\right)$ is homotopy equivalent to a six point space (corresponding to the six dihomotopy classes of dipaths pictured in 2), and that $\overrightarrow{\mathfrak{T}}(Y)\left(\alpha_{Y}, \beta_{Y}\right)$ is also homotopy equivalent to a six point space (corresponding again to the six dihomotopy classes of dipaths pictured in Figure 2).

It can be shown that those two programs are not equivalent in the sense that they do not act the same way. But their trace spaces have the homotopy type, the one of a discrete space with six points.

Our main idea is then to see how homotopy type of trace spaces vary when we move the points between which we take the traces.

With the possibility to consider all the trace spaces, we can distinguish the two former pospaces because if we consider the trace space between $\alpha$ and $\beta$, as in Figure 3, it has the homotopy type of a discrete space with four points but we can show that there is no pair of points between which we have a trace space with the same homotopy type.

Let $X$ be a pospace, we first define the "base" category on which to make vary the end points of traces, and on top of which we will observe the variation of topology of trace spaces. 
Definition 2.5 We define $\mathcal{T}_{X}$ the category whose:

- objects are traces of $X$

- morphisms (also called extensions) from $\langle p\rangle$ to $\langle q\rangle$ with $p$ dipath from $x$ to $y$ and $q$ from $x^{\prime}$ to $y^{\prime}$ are pairs of traces $(\langle\alpha\rangle,\langle\beta\rangle)$ such that $\langle q\rangle=\langle\alpha \star p \star \beta\rangle$

We then define $\vec{T}_{*}(X): \mathcal{T}_{X} \longrightarrow \boldsymbol{T o p}_{*}$ which maps :

- every trace $\langle p\rangle$ with $p$ from $x$ to $y$ to the pointed space $(\overrightarrow{\mathfrak{T}}(X)(x, y),\langle p\rangle)$

- every extension $(\langle\alpha\rangle,\langle\beta\rangle)$ with $\alpha$ dipath from $x^{\prime}$ to $x$ and $\beta$ dipath from $y$ to $y^{\prime}$ to the continuous map $\left\langle\alpha \star_{-} \star \beta\right\rangle: \overrightarrow{\mathfrak{T}}(X)(x, y) \longrightarrow \overrightarrow{\mathfrak{T}}(X)\left(x^{\prime}, y^{\prime}\right)$ which maps $\langle p\rangle$ to $\langle\alpha \star p \star \beta\rangle$.

Diagrams in a category form themselves a category, which we make explicit below :

Definition 2.6 (The category of diagrams) Let $\mathcal{M}$ be a category. We define $\operatorname{Diag}(\mathcal{M})$ the category whose :

- objects are diagrams i.e. functors from any small category $\mathcal{C}$ to $\mathcal{M}$

- morphisms from $F: \mathcal{C} \longrightarrow \mathcal{M}$ to $G: \mathcal{D} \longrightarrow \mathcal{M}$ are pairs $(\Phi, \sigma)$ where

- $\Phi$ is a functor from $\mathcal{C}$ to $\mathcal{D}$

- $\sigma$ is a natural transformation from $F$ to $G \circ \Phi$

- the identity on $F: \mathcal{C} \longrightarrow \mathcal{M}$ is $i d_{F}=\left(i d_{\mathcal{C}}, 1_{F}\right)$ where

- $i d_{C}$ is the identity functor on $\mathcal{C}$

$-1_{F}$ is the identity natural transformation on $F$

- the composition is defined as follows : $(\Psi, \tau) \circ(\Phi, \sigma)$ where $(\Phi, \sigma):(F$ : $\mathcal{C} \longrightarrow \mathcal{M}) \longrightarrow(G: \mathcal{D} \longrightarrow \mathcal{M})$ and $(\Psi, \tau):(G: \mathcal{D} \longrightarrow \mathcal{M}) \longrightarrow(H:$ $\mathcal{E} \longrightarrow \mathcal{M})$ is $\left(\Psi \circ \Phi,\left(\tau_{\Phi(c)} \circ \sigma_{c}\right)_{c \in O b(\mathcal{M})}\right)$

Now, trace diagrams can be seen as a functor into diagrams in $\mathbf{T o p}_{*}$ :

Proposition $2.7 \vec{T}$ extends to a functor from PoTop to Diag $\left(\boldsymbol{T o p}_{*}\right)$.

Proof.

If $f: X \longrightarrow Y$ is a dimap, we define $\vec{T}(f)=(\Phi, \sigma): \vec{T}(X) \longrightarrow \vec{T}(Y)$ this way :

- $\Phi: \mathcal{T}_{X} \longrightarrow \mathcal{T}_{Y}$ such that $\Phi(\langle p\rangle)=\langle f \circ p\rangle$ and $\Phi(\langle\alpha\rangle,\langle\beta\rangle)=(\langle f \circ \alpha\rangle,\langle f \circ \beta\rangle)$

- if $p$ is a dipath from $x$ to $y, \sigma_{\langle p\rangle}: \overrightarrow{\mathfrak{T}}(X)(x, y) \longrightarrow \overrightarrow{\mathfrak{T}}(Y)(f(x), f(y))$ $\langle p\rangle \mapsto\langle f \circ p\rangle$ 
From trace diagrams in $\mathbf{T o p}_{*}$ we can derive relevant homological and homotopical information by composing with the classical singular homology (resp. homotopy) functor in the category $\mathrm{Ab}$ of abelian groups (resp. Grp)

Definition 2.8 (Natural homotopy) We define for $n \geq 1, \vec{\Pi}_{n}(X): \mathcal{T}_{X} \longrightarrow$ $\mathcal{M}$ (where $\mathcal{M}$ is either $\boldsymbol{S e t}, \boldsymbol{G r p}$ or $\boldsymbol{A b}$ ) composing $\vec{T}_{*}(X)$ with the functor which maps its $n-1$ th homotopy group (set if $n=1$ ).

Definition 2.9 (Natural homology) We define for $n \geq 1, \vec{H}_{n}(X): \mathcal{T}_{X} \longrightarrow$ $\mathcal{M}$ (where $\mathcal{M}$ is $\boldsymbol{A b}$ ) composing $\vec{T}_{*}(X)$ with the functor which maps its $n-1$ th homology group.

Remark 2.10 $\mathcal{T}_{X}$ is actually the category of factorization (or twisted arrow category [34]) of the category whose objects are points of $X$ and morphisms are traces which makes $\vec{T}_{*}(X)$ a natural system [1].

Example 2.3 We consider the following pospace, noted $a+b$, which is composed of copy of two directed segments a and $b$ where there initial points are identified, and their final points are identified too. In the following picture, we distinguish two particular points $x$ and $y$ on $a$, with $x<y$ (respectively $x^{\prime}$ and $y^{\prime}$ on $b$, with $\left.x^{\prime}<y^{\prime}\right)$, which we will use to describe the category of factorization $\mathcal{T}_{a+b}$ as well as the natural homology $\vec{H}_{n}(a+b)$.

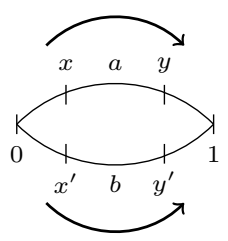

The description of $\mathcal{T}_{a+b}$ is now as follows. Objects of $\mathcal{T}_{a+b}$ are dipaths, which can be either :

- constant dipaths, $0, x, y, x^{\prime}, y^{\prime}, 1$, for all points $x, y, x^{\prime}, y^{\prime}$ that we chose to distinguish in the picture of $a+b$.

- non constant and non maximal dipaths of the form $[0, x],[x, y],[y, 1]$ etc.

- maximal dipaths $a$ and $b$

We chose below to draw a picture of a subcategory of $\mathcal{T}_{a+b}$, where $x, y$, $x^{\prime}$ and $y^{\prime}$ are any distinguished points of $a$ and $b$ as discussed before. The extension morphisms in $\mathcal{T}_{a+b}$ are pictured below as arrows; for instance, there is an extension morphism from dipath $[x, y]$ to $[0, y]$ and to $[x, 1]$, among other extension morphisms : 


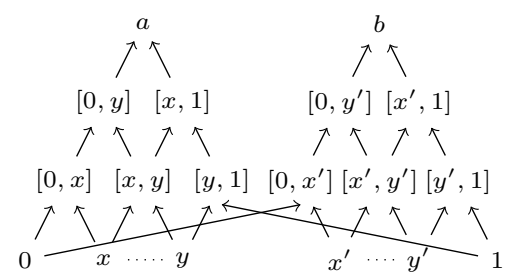

Now, we can picture a subdiagram of $\vec{H}_{1}(a+b)$, by applying the homology functor on the trace spaces from the starting point to the end point of the dipaths, objects of $\mathcal{T}_{a+b}$. For instance, the trace space $\overrightarrow{\mathfrak{T}}(a+b)(x, y)$ (respectively $\overrightarrow{\mathfrak{T}}(a+b)(0, y))$ corresponding to dipath $[x, y]$ (respectively $[0, y]$ ) in the diagram above, is just a point, hence has zeroth homology group equal to $\mathbb{Z}$ (respectively $\mathbb{Z}$ ). All other zeroth homology groups are trivial at the exception of the ones corresponding to the two maximal dipaths (up to reparametrization) a and $b$, going from 0 to 1 . In that case, $\overrightarrow{\mathfrak{T}}(a+b)(0,1)$ is composed of two points, that we can identify with $a$ and $b$, and has $\mathbb{Z}^{2}$ (or $\mathbb{Z}[a, b]$ with the identification we just made) as zeroth homology. Now the extension morphism from $[0, y]$ to a induces a map in homology which maps the only generator of $H_{0}(\overrightarrow{\mathfrak{T}}(a+b)(0, y))$ to generator a in $\mathbb{Z}[a, b]$ as indicated in the picture below :

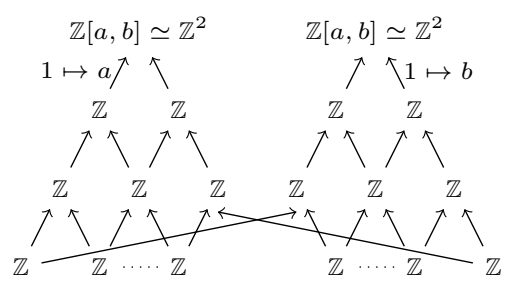

\subsection{Pre-cubical sets, cubical complexes}

Now, we turn to an alternative definition of natural homology and homotopy, on a combinatorial version of pospaces. As in the classical case, we want to express that our combinatorial and topological homology and homotopy theories are strongly related. This will be Theorem 3.6.

Definition 2.11 $A$ pre-cubical set $K$ is a graded set $\left(K_{n}\right)_{n \in \mathbb{N}}$ together with (face) maps $\partial_{i}^{\alpha}: K_{n} \longrightarrow K_{n-1}$ with $1 \leq i \leq n$ and $\alpha \in\{0,1\}$ satisfying :

$$
\partial_{i}^{\alpha} \circ \partial_{j}^{\beta}=\partial_{j-1}^{\beta} \circ \partial_{i}^{\alpha}
$$

In order to relate (some) pre-cubical sets with pospaces, we need to define a geometric realization, hence first, topological "models" for abstract $n$-cubes :

Definition 2.12 Let $\square_{n}=\left\{\left(t_{1}, \ldots, t_{n}\right) \in \mathbb{R}^{n} \mid \forall 1 \leq i \leq n, 0 \leq t_{i} \leq 1\right\}$ the standard $n$-cube in $\mathbb{R}^{n}$. 
We define for $\alpha \in\{0,1\}, n \in \mathbb{N}, 1 \leq i \leq n+1, \rho_{i}^{\alpha}: \square_{n} \rightarrow \square_{n+1}$ by :

$$
\rho_{i}^{\alpha}\left(t_{1}, \ldots, t_{n}\right)=\left(t_{1}, \ldots, t_{i-1}, \alpha, t_{i}, \ldots, t_{n}\right)
$$

Let $K$ be a pre-cubical set. We note $R(K)=\bigsqcup_{n \in \mathbb{N}} K_{n} \times \square_{n}$, the topological space constructed as the disjoint union (with disjoint topology) of the product (with the product topology) of $K_{n}$ (with the discrete topology) and $\square_{n}$ (with the topology of $\mathbb{R}^{n}$ ).

The elements of $R(K)$ are pairs $(e, \vec{a})$ where $e$ is an $n$-dimensional cube in $K$ and $\vec{a} \in[0,1]^{n}$, for some $n$.

The geometric realization Geom $(K)$ of $K$ is the quotient of $R(K)$ by the least equivalence relation $\equiv$ such that :

$\forall \alpha \in\{0,1\}, n \in \mathbb{N}, 1 \leq i \leq n, x \in K_{n}, t \in \square_{n-1},\left(\partial_{i}^{\alpha}(x), t\right) \equiv\left(x, \rho_{i}^{\alpha}(t)\right)$

with the quotient topology.

We shall write $[e, \vec{a}]$ for the point obtained as the equivalence class of $(e, \vec{a})$.

Geometric realizations of pre-cubical sets are directed spaces of a much more general type than the pospaces we have been considering up to now. We restrict our study to particular "geometric" complexes, which are realizations of specific pre-cubical sets and form pospaces :

Definition 2.13 $A$ (d-dimensional) cubical complex $K$ is a finite set of cubes $(D, \vec{x})$, where $D \subseteq\{1,2, \cdots, d\}$ and $\vec{x} \in \mathbb{Z}^{d}$, which is closed under taking past and future faces (to be defined below). The cardinality $|D|$ of $D$ is the dimension of the cube $(D, \vec{x})$. Let $\overrightarrow{1}_{k}$ be the d-tuple whose $k$ th component is 1 , all others being 0 . Each cube $(D, \vec{x})$ is realized as the geometric cube $\iota(D, \vec{x})=I_{1} \times I_{2} \times$ $\cdots \times I_{d}$ where $I_{k}=\left[x_{k}, x_{k}+1\right]$ if $k \in D, I_{k}=\left[x_{k}, x_{k}\right]$ otherwise, matching the definition of [30].

When $|D|=n$, we write $D[i]$ for the ith element of $D$. For example, if $D=\{3,4,7\}$, then $D[1]=3, D[2]=4, D[3]=7$. We also write $\partial_{i} D$ for $D$ minus $D[i]$. Every $n$-dimensional cube $(D, \vec{x})$ has $n$ past faces $\partial_{i}^{0}(D, \vec{x})$, defined as $\left(\partial_{i} D, x\right)$, and $n$ future faces $\partial_{i}^{1}(D, \vec{x})$, defined as $\left(\partial_{i} D, x+\overrightarrow{1}_{D[i]}\right), 1 \leq i \leq n$.

Proposition 2.14 A cubical complex is a pre-cubical set. Moreover,

$$
\begin{aligned}
\epsilon: \quad \operatorname{Geom}(K) & \rightarrow \underset{(D, \vec{x}) \in K}{\bigsqcup} \iota(D, \vec{x}) \\
{[(D, \vec{x}), a] } & \mapsto \vec{x}+\sum_{i=1}^{n} a_{i} \overrightarrow{1}_{D[i]}
\end{aligned}
$$

is a homeomorphism and thus induces a pospace structure on $\operatorname{Geom}(K)$ by $[(D, \vec{x}), a] \leq\left[\left(D^{\prime}, \vec{x}^{\prime}\right), a^{\prime}\right]$ iff $\epsilon([(D, \vec{x}), a]) \leq \epsilon([(D, \vec{x}), a])$. We note $\overrightarrow{G e o m}(K)$ this pospace. 


\subsection{Combinatorial natural homology}

Let $K$ be a pre-cubical set. In this section, we define the analogous of the (continuous) traces in the (discrete) setting of pre-cubical set, so that to build a factorization category (Definition 2.17) and then a natural homology (Definition $2.18)$.

Definition 2.15 Let $x$ and $y \in K$. We say that $x$ is a future boundary (resp. $a$ past boundary) of $y$ if there exist $k \geq 0$ and $i_{0}, \ldots, i_{k}$ such that $x=\partial_{i_{k}}^{1} \circ \cdots \circ$ $\partial_{i_{0}}^{1}(y)$ (resp. $x=\partial_{i_{k}}^{0} \circ \cdots \circ \partial_{i_{0}}^{0}(y)$ ). We note $x \preceq y$ when :

- either $x$ is a past boundary of $y$

- either $y$ is a future boundary of $x$

Definition 2.16 $A$ discrete trace from $x$ to $y$ in $K$ is a sequence $c_{0}, \ldots, c_{n}$ of $K$ (with $n \geq 0$ ) such that $c_{0}=x, c_{n}=y$ and for all $i \in\{1, \ldots, n\} c_{i-1} \preceq c_{i}$.

Similarly as for pospaces (Definition 2.5), discrete traces of a pre-cubical set $K$ form a category $\mathcal{T}_{K}^{d}$ whose objects are discrete traces, and whose morphisms are extensions of discrete traces which are discrete traces.

We note first that when $K$ is a cubical complex, a discrete trace can be realized as a trace in the geometric realization $\overrightarrow{\operatorname{Geom}}(K)$. For $x$ in $K$, write $\hat{x}$ the point $[x, \bullet]$ of $\operatorname{Geom}(K)$ where $\bullet=\left(\frac{1}{2}, \ldots, \frac{1}{2}\right)$. When $x$ is a past boundary of $y$, i.e. $x=\partial_{i_{k}}^{0} \circ \cdots \circ \partial_{i_{0}}^{0}(y)$, write $\widehat{x y}$ the path in $\operatorname{Geom}(K)$ from $\hat{x}$ to $\hat{y}$ defined by

$$
\widehat{x y}(t)=\left[y, t \bullet+(1-t) \rho_{i_{0}}^{0} \circ \ldots \circ \rho_{i_{k}}^{0}(\bullet)\right]
$$

When $y$ is a future boundary of $x$, i.e. $y=\partial_{i_{k}}^{1} \circ \cdots \circ \partial_{i_{0}}^{1}(x)$, write $\widehat{x y}$ the path in $\operatorname{Geom}(K)$ from $\hat{x}$ to $\hat{y}$ defined by

$$
\widehat{x y}(t)=\left[x,(1-t) \bullet+t \rho_{i_{0}}^{1} \circ \ldots \circ \rho_{i_{k}}^{1}(\bullet)\right]
$$

$\widehat{x y}$ is uniquely defined when $K$ is a non-self linked pre-cubical set [12] and is a dipath when $K$ is a cubical complex.

Then, a discrete trace $c_{0}, \ldots, c_{n}$ can be realized as the trace $\left\langle\widehat{c_{0} c_{1}} \star \ldots \star \widehat{c_{n-1} c_{n}}\right\rangle$ in $\overrightarrow{G e o m}(K)$, noted $\widehat{c_{0}, \ldots, c_{n}}$.

Definition 2.17 We define $\mathcal{T}_{K}^{d}$ the category whose :

- objects are discrete traces of $K$

- morphisms from $c_{0}, \ldots, c_{n}$ (discrete trace from $x$ to y) to $d_{0}, \ldots, d_{m}$ (discrete trace from $x^{\prime}$ to $\left.y^{\prime}\right)$ are pairs of discrete traces $\left(\left(e_{0}, \ldots, e_{p}\right),\left(f_{0}, \ldots, f_{k}\right)\right)$ from $\left(x^{\prime}, y\right)$ to $\left(x, y^{\prime}\right)$ (so $c_{0}=e_{p}=x$ and $c_{n}=f_{0}=y$ ) such that $d_{0}, \ldots, d_{m}=e_{0}, \ldots, e_{p-1}, c_{0}, \ldots, c_{n}, f_{1}, \ldots, f_{k}$.

Then $\widehat{ }$ extends to a functor from $\mathcal{T}_{K}^{d}$ to $\mathcal{T}_{\overrightarrow{\operatorname{Geom}}(K)}$. 
Definition 2.18 Let $K$ be a cubical complex. For $n \geq 1$, we define the $n$-th natural homology of $K$ or discrete natural homology $\vec{h}_{n}(K): \mathcal{T}_{K}^{d} \longrightarrow \boldsymbol{A b}$ as

$$
\vec{h}_{n}(K)=\vec{H}_{n}(\overrightarrow{\operatorname{Geom}}(K)) \circ \text {. }
$$

But natural homology in the combinatorial version do not quite correspond to natural homology in the topological case, as we exemplify now :

Example 2.4 We continue with Example 2.3. The space we called $a+b$ is dihomeomorphic to the digeometric realization of the following 2-dimensional cubical complex $K_{a+b}$ :

$$
\begin{gathered}
\left\{\alpha=(\varnothing,(0,0)), \beta_{1}=(\varnothing,(0,1)), \beta_{2}=(\varnothing,(1,0)), \gamma=(\varnothing,(1,1)),\right. \\
\left.a_{1}=(\{2\},(0,0)), b_{1}=(\{1\},(0,1)), a_{2}=(\{1\},(0,0)), b_{2}=(\{2\},(1,0))\right\}
\end{gathered}
$$

which can be geometrically depicted like this :

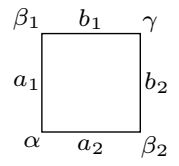

The category $\mathcal{T}_{K_{a+b}}^{d}$ is :

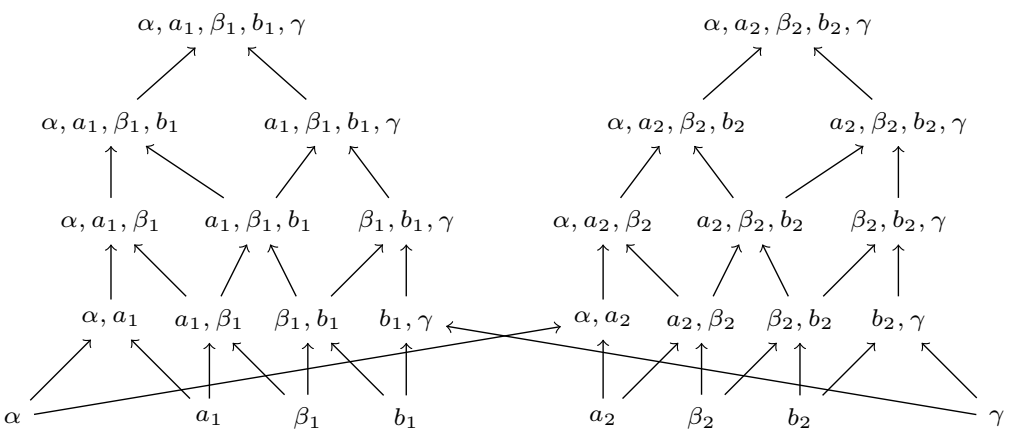

The image of $\vec{h}_{1}\left(K_{a+b}\right)$ is :

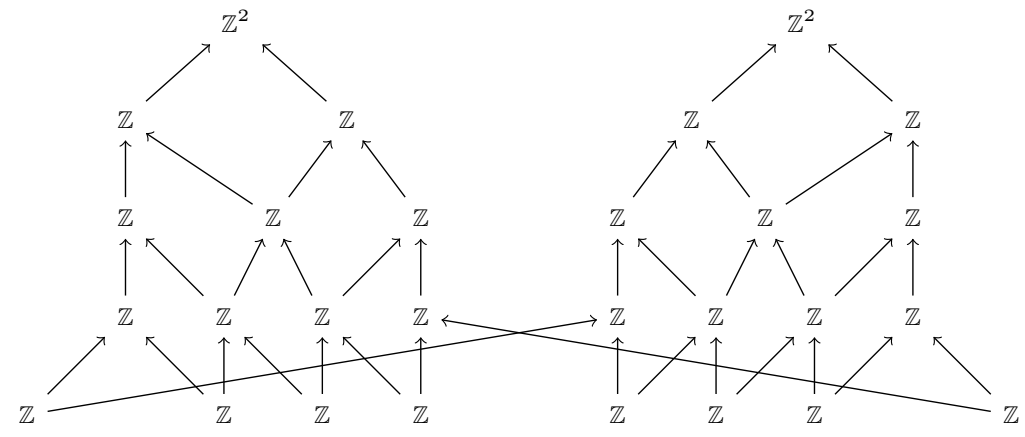


Still, the difference between this (discrete) natural homology with what we had in the continuous case, in Example 2.3, is somehow inessential. We now define an weaker equivalence than isomophism of diagrams that abstract away this difference. This is based on the notion of bisimulation, which has been introduced in concurrency theory [41,37]. We will see in Section 3.1 that this equivalence can be seen as a bisimulation equivalence defined by a notion of open maps [29].

\section{Bisimulation of diagrams}

\section{1 $\mathcal{P}$-bisimilarity of diagrams}

First, we recap the general construction of bisimulations with open maps [29], we then apply it to our category of diagrams.

Definition 3.1 Given a category $\mathcal{N}$ and a subcategory $\mathcal{P}$ (called category of paths), we say that a morphism $f: X \longrightarrow Y$ of $\mathcal{N}$ is $\mathcal{P}$-open if for every diagram :

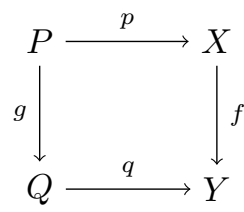

where $g: P \longrightarrow Q$ is a morphism of $\mathcal{P}$, then there exists $r: Q \longrightarrow X$ making the following diagram commutative:

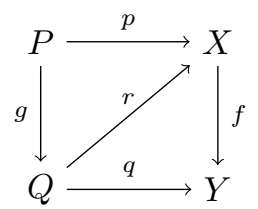

In other words, $f$ has the right lifting property with respect to morphisms of $\mathcal{P}$. We then say that two objects $X$ and $Z$ of $\mathcal{N}$ are $\mathcal{P}$-bisimilar if there exists a span of open maps between them :

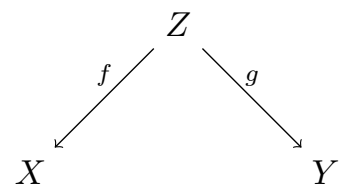

We consider now the category $\mathcal{N}=\operatorname{Diag}_{\text {iso }}(\mathcal{M})$, which is the subcategory of $\operatorname{Diag}(\mathcal{M})$ whose morphisms are of the form $(\Phi, \sigma)$ where $\sigma$ is a natural 
isomorphism. We consider $\mathcal{P}$ the following subcategory of $\mathcal{N}$ : its objects are the functors of $\mathbf{n}$ in $\mathcal{M}$ for any $n \in \mathbb{N}$ (we will call them path of length $n$ ) where $\mathbf{n}$ is the category induced by the natural total order on $\{1, \ldots, n\}$, i.e. :

$$
1 \longrightarrow 2 \longrightarrow \cdots \longrightarrow \mathrm{n}
$$

and its morphisms are the morphisms of $\operatorname{Diag}_{i s o}(\mathcal{M})$ of the form $\left(e x t_{n, m}, i d\right)$ with $n \leq m$ where $\operatorname{ext}_{n, m}: \mathbf{n} \longrightarrow \mathbf{m}$ is the functor induced by the increasing function $(i \mapsto i)$. We will also note those morphisms $\operatorname{ext}_{n, m}$.

Lemma 3.2 A morphism $(\Phi, \sigma):(F: \mathcal{C} \longrightarrow \mathcal{M}) \longrightarrow(G: \mathcal{D} \longrightarrow \mathcal{M}) \in$ $\operatorname{Diag}_{\text {iso }}(\mathcal{M})$ is $\mathcal{P}$-open iff it has the right lifting property for ext ${ }_{n, n+1}$ for all $n \in \mathbb{N}$.

\section{Proof.}

$\Rightarrow$ by definition of open maps

$\Leftarrow$ we show that it has the right lifting property with respect to $e x t_{n, m}$, using the fact that $e x t_{n, m}=e x t_{m-1, m} \circ \ldots \circ e x t_{n, n+1}$.

\subsection{Equivalent definitions of $\mathcal{P}$-bisimilarity of diagrams}

This section is not required, as a first read, for proving the main results of this section, in particular equivalence under bisimulation of combinatorial and topological homology theories, Section 3.3. But they shed an interesting light on this notion of equivalence, that readers might find closer to their culture.

\subsubsection{Via a simpler notion of open maps}

Proposition 3.3 A morphism $(\Phi, \sigma):(F: \mathcal{C} \longrightarrow \mathcal{M}) \longrightarrow(G: \mathcal{D} \longrightarrow \mathcal{M}) \in$ $\operatorname{Diag}_{\text {iso }}(\mathcal{M})$ is $\mathcal{P}$-open iff it has the right lifting property for ext $t_{0,1}$ and ext $t_{1,2}$ i.e. iff :

- $\Phi$ is surjective on objects

- for every morphism $j: d \longrightarrow d^{\prime}$ of $\mathcal{D}$ and every $c$ of $\mathcal{C}$ such that $\Phi(c)=d$ there exists $i: c \longrightarrow c^{\prime}$ such that $\Phi(i)=j$.

\section{Proof.}

$\Rightarrow$ by definition of open maps

$\Leftarrow$ Let us show that $(\Phi, \sigma)$ as the right lifting property with respect to $e t_{n, n+1}$. The case 0 is by hypothesis. Assume $n \geq 1$ and given a commutative diagram : 


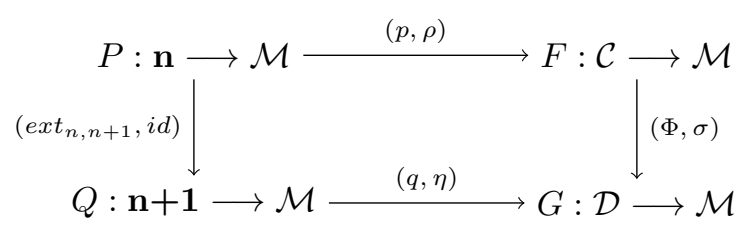

We want $(r, \theta)$ such that :

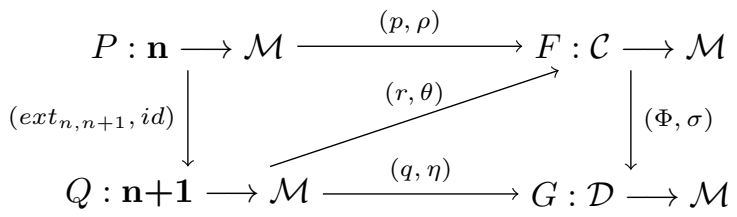

commutes.

$r: \mathbf{n}+\mathbf{1} \longrightarrow \mathcal{C}$ is constructed this way :

- for $1 \leq i \leq n, r(i)=p(i)$ and for all $j \leq i, r(j \leq i)=p(j \leq i)$

- it remains to construct $r(n \leq n+1)$ (which will determine $r(n+1)$ ). We know that $q(n \leq n+1): q(n) \longrightarrow q(n+1)$ and that $q(n)=$ $\Phi(p(n))$. Thus by the second property of $\Phi$, there exists a morphism $i: p(n)=r(n) \longrightarrow c$ of $\mathcal{C}$ such that $\Phi(i)=q(n \leq n+1)$. We pose $r(n \leq n+1)=i$.

First, it is easy to see that $p=r \circ e^{2 x} t_{n, n+1}$ and $\Phi \circ r=q$.

$\theta=\left(\theta_{i}\right)_{i \leq n+1}: Q \longrightarrow F \circ r$ is define this way :

- for all $i \leq n, \theta_{i}=\rho_{i}=\sigma_{q(i)} \circ \eta_{i}$ which is an isomorphism

$-\theta_{n+1}=\sigma_{q(n+1)} \circ \eta_{n+1}$ which is an isomorphism

$\theta$ is natural because it is the composition of two natural transformations. By construction, it makes the diagram commutes.

By abuse of notation, we will say that a morphism $(\Phi, \sigma)$ of $\operatorname{Diag}(\mathcal{M})$ is open if $\sigma$ is a natural isomorphism and $\Phi$ satisfies the conditions of the previous proposition.

\subsubsection{A relational characterization of bisimilarity}

We now turn to a more classical characterization of bisimulation for our diagrams, that relates to theoretical computer science and concurrency theory :

Definition $3.4 A$ bisimulation $R$ between two diagrams $F: \mathcal{C} \longrightarrow \mathcal{M}$ and $G: \mathcal{D} \longrightarrow \mathcal{M}$ is a set of triples $(c, f, d)$ where $c$ is an object of $\mathcal{C}, d$ is an object of $\mathcal{D}$ and $f: F(c) \longrightarrow G(d)$ is an isomorphism of $\mathcal{M}$ such that for all $(c, f, d)$ in $R$ : 
- if there exists $i: c \longrightarrow c^{\prime} \in \mathcal{C}$ then there exists $j: d \longrightarrow d^{\prime} \in \mathcal{D}$ and $g: F\left(c^{\prime}\right) \longrightarrow G\left(d^{\prime}\right) \in \mathcal{M}$ such that $g \circ F(i)=G(j) \circ f$ and $\left(c^{\prime}, g, d^{\prime}\right) \in R$

- if there exists $j: d \longrightarrow d^{\prime} \in \mathcal{D}$ then there exists $i: c \longrightarrow c^{\prime} \in \mathcal{C}$ and $g: F\left(c^{\prime}\right) \longrightarrow G\left(d^{\prime}\right) \in \mathcal{M}$ such that $g \circ F(i)=G(j) \circ f$ and $\left(c^{\prime}, g, d^{\prime}\right) \in R$

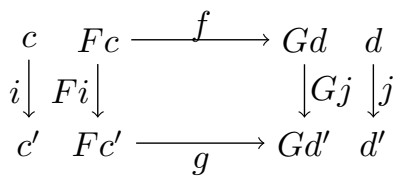

and such that :

- for all $c \in \mathcal{C}$, there exists $d$ and $f$ such that $(c, f, d) \in R$

- for all $d \in \mathcal{D}$, there exists $c$ and $f$ such that $(c, f, s) \in R$

Proposition 3.5 Two functors $F: \mathcal{C} \longrightarrow \mathcal{M}$ and $G: \mathcal{D} \longrightarrow \mathcal{M}$ are $\mathcal{P}$-bisimilar iff there exists a bisimulation between them.

\section{Proof.}

$\Rightarrow$ Assume that there is a span :

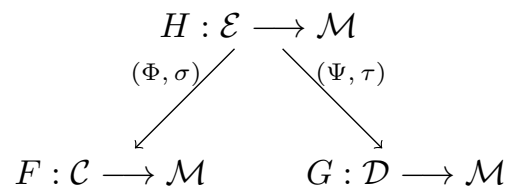

of open maps (here in the sense of the previous proposition). We define $R=\left\{\left(\Phi(e), \tau_{e} \circ \sigma_{\Phi(e)}^{-1}, \Psi_{e}\right) \mid e \in \mathcal{E}\right\}$ and show that this is a bisimulation. First, it is well defined because $\tau$ and $\sigma$ are isomorphisms. The third condition of a bisimulation comes from the surjectivity of $\Phi$. Idem for the forth and the surjectivity of $\Psi$. The first condition comes from the second condition on $\Phi$ of an open map : let $\left(\Phi(e), \tau_{e} \circ \sigma_{\Phi(e)}^{-1}, \Psi_{e}\right)$ in $R$ and $i: \Phi(e) \longrightarrow c^{\prime} \in \mathcal{C}$. By the condition on $\Phi$ there exists $k: e \longrightarrow e^{\prime}$ in $\mathcal{E}$ such that $\Phi(k)=i$. Then define $j=\Psi(k), d^{\prime}=\Psi\left(e^{\prime}\right)$ and $g=\tau_{e^{\prime}} \circ \sigma_{\Phi\left(e^{\prime}\right)}^{-1}$. $\left(\Phi\left(e^{\prime}\right), g, d^{\prime}\right)$ belongs to $R$ by construction and $g \circ F(i)=G(j) \circ \tau_{e} \circ \sigma_{\Phi(e)}^{-1}$ by naturality of $\sigma$ and $\tau$. Idem for the second condition of a bisimulation.

$\Leftarrow$ Assume now that there is a bisimulation $R$ between $F$ and $G$. We will construct a span of open maps. Let $\mathcal{E}$ be the small category whose objects are elements of $R$, and whose morphisms from $(c, f, d)$ to $\left(c^{\prime}, f^{\prime}, d^{\prime}\right)$ are pairs $(i, j)$ of a morphism $i: c \longrightarrow c^{\prime}$ in $\mathcal{C}$ and of a morphism $j: d \longrightarrow d^{\prime}$ in $\mathcal{D}$, such that the following diagram commutes: 


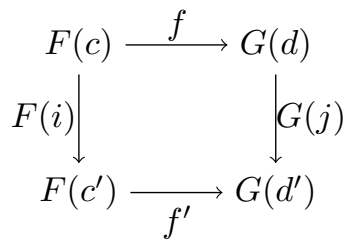

Define the tip $H$ of the span between $F$ and $G$ as the functor $H: E \longrightarrow \mathcal{M}$ that maps every object $(c, f, d) \in R$ to $F(c)$, and every morphism $(i, j)$ : $(c, f, d) \longrightarrow\left(c^{\prime}, f^{\prime}, d^{\prime}\right)$ to $F(i): F(c) \longrightarrow F\left(c^{\prime}\right)$.

We now build a morphism $(\Phi, \sigma)$ from $H$ to $F$. We start by building $\Phi: \mathcal{E} \longrightarrow \mathcal{C}$. We define $\Phi$ as the functor that maps every object $(c, f, d)$ to $c$ and every morphism $(i, j):(c, f, d) \longrightarrow\left(c^{\prime}, f^{\prime}, d^{\prime}\right)$ to $i: c \longrightarrow c^{\prime}$. We verify that $\Phi$ satisfies the condition of the previous proposition :

1. $\Phi$ is surjective on objects: this is third condition of the definition of $R$ as a bisimulation.

2. Let $i: \Phi(e) \longrightarrow c^{\prime}$ be a morphism of $X$. The object $e$ must be a triple $(c, f, d) \in R$, and $i$ is a morphism from $c$ to $c^{\prime}$ in $\mathcal{C}$. By the first condition of the definition of $R$ as a bisimulation, there is a triple $\left(c^{\prime}, f^{\prime}, d^{\prime}\right) \in R$ and a morphism $j: d \longrightarrow d^{\prime}$ of $\mathcal{D}$ such that the following diagram commutes:

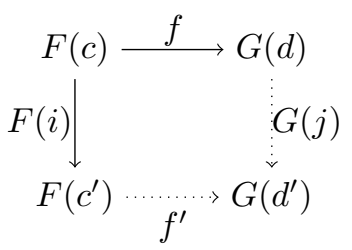

In particular, $(i, j)$ is a morphism of $E$, from $(c, f, d)$ to $\left(c^{\prime}, f^{\prime}, d^{\prime}\right)$. Moreover, $H(i, j)=i$.

For every $(c, f, d) \in R$, let $\sigma_{(c, f, d)}=i d_{F(c)}: H(c, f, d)=F(c) \longrightarrow$ $F \circ \Phi(c, f, d)=F(c)$. Those are isomorphisms, and define a natural transformation $\sigma: H \longrightarrow F \circ \Phi$. It follows that $(\Phi, \sigma)$ is an open map from $H$ to $F$.

We define the open map $(\Psi, \tau)$ from $H$ to $G$ similarly.

\subsection{Combinatorial and topological homology theories are bisimilar}

We are now in a position to prove that, at least for cubical complexes, the homology theories we have defined combinatorially, and topologically, are equivalent. This is to be regarded as similar to the equivalence between simplicial homology and singular homology composed with geometric realization : 
Theorem 3.6 If $K$ is a cubical complex, then there exists an open map

$$
(\mathcal{C}, \sigma): \vec{H}_{n}(\overrightarrow{\operatorname{Geom}}(K)) \longrightarrow \vec{h}_{n}(K)
$$

In particular $\vec{H}_{n}(\overrightarrow{G e o m}(K))$ and $\vec{h}_{n}(K)$ are bisimilar.

First, let us define $\mathcal{C}: \mathcal{T}_{\overline{\operatorname{Geom}(K)}} \longrightarrow \mathcal{T}_{K}$. If $b$ is a point of $\overrightarrow{\operatorname{Geom}}(K)$, it can uniquely be written as $[e, \vec{a}]$ where $a \in] 0,1\left[{ }^{k}\right.$ for a certain $k$. We note $\mathcal{C}(b)=e \in K$ and call it carrier of $b$. For a dipath, we can intuitively define the sequence of all the cube that are the carrier of a point of this dipath. Formally,

Proposition 3.7 ([11]) Given a dipath $\pi$ of $\overrightarrow{G e o m}(K)$, there is a unique sequence $c_{0}, c_{1}, \cdots, c_{k}$ of elements of $K$ and a unique sequence of real numbers $0=t_{0} \leq t_{1} \leq \cdots \leq t_{k} \leq t_{k+1}=1$ (call them the times of change) such that:

- for every $1 \leq i \leq k, c_{i-1} \neq c_{i}$,

- for every $0 \leq i \leq k$, for every $t \in\left[t_{i}, t_{i+1}\right], \pi(t)$ is a point of the form $[c, \vec{a}]$ with $c=c_{i}$,

- for every $0 \leq i \leq k$, for every $t \in\left(t_{i}, t_{i+1}\right), \mathcal{C}(\pi(t))=c_{i}$,

- $\mathcal{C}(\pi(0))=c_{0}$ and $\mathcal{C}(\pi(1))=c_{k}$,

- for every $1 \leq i \leq k, \mathcal{C}\left(\pi\left(t_{i}\right)\right) \in\left\{c_{i-1}, c_{i}\right\}$ and if furthermore $t_{i}=t_{i+1}$ then $\mathcal{C}\left(\pi\left(t_{i}\right)\right)=c_{i}$.

The sequence $c_{0}, c_{1}, \cdots, c_{k}$ is a discrete trace and is called the carrier sequence of $\pi$. Two dipaths that are equivalent modulo reparametrization have the same carrier sequence, so it is legitimate to call carrier sequence of a trace $\langle\pi\rangle$ the carrier sequence $\mathcal{C}(\pi)$ of $\pi$.

This induces a functor $\mathcal{C}: \mathcal{T}_{\overrightarrow{\operatorname{Geom}(K)}} \longrightarrow \mathcal{T}_{K}$ which have the property of the functorial part of an open map. For the lifting property, this a consequence of the following lemma :

Lemma 3.8 Let $\langle\pi\rangle$ be a trace in $\overrightarrow{G e o m}(K)$ with carrier sequence $c_{0} \preceq c_{1} \preceq$ $\cdots \preceq c_{k}$.

- For every cube $c_{-1} \preceq c_{0}$, there is a dipath $\alpha$ in $X$ such that $\mathcal{C}(\langle\alpha \star \pi\rangle)=$ $c_{-1} \preceq c_{0} \preceq c_{1} \preceq \cdots \preceq c_{k}$.

- For every cube $c_{k+1}$ such that $c_{k} \preceq c_{k+1}$, there is a dipath $\beta$ in $X$ such that $\mathcal{C}(\langle\pi \star \beta\rangle)=c_{0} \preceq c_{1} \preceq \cdots \preceq c_{k} \preceq c_{k+1}$.

Proof. We examine the second case only: the other case is symmetric. Since $c_{k} \preceq c_{k+1}, c_{k}$ can be a past boundary of $c_{k+1}$, or $c_{k+1}$ can be a future boundary of $c_{k}$. We examine both cases: 
- If $c_{k}$ is a past boundary of $c_{k+1}$, say $c_{k}=\partial_{i_{p}}^{0} \cdots \partial_{i_{0}}^{0} c_{k+1}$, then by using the precubical equations we may require $i_{0}>\ldots>i_{p}$. Writing $\pi(1)$ as $\left[c_{k}, \vec{a}\right]$, we also have $\pi(1)=\left[c_{k+1}, \delta_{i_{0}}^{0} \cdots \delta_{i_{p}}^{0} \vec{a}\right]$ by the definition of the geometric realization. Since $\mathcal{C}(\pi(1))=c_{k}$, no component $a_{i}$ of $\vec{a}$ is equal to 0 or 1. Let $\vec{b}=\delta_{i_{0}}^{0} \cdots \delta_{i_{p}}^{0} \vec{a}$ : it follows that the components $b_{i}$ of $\vec{b}$ that are equal to 0 are exactly those such that $i \in\left\{i_{0}, \cdots, i_{p}\right\}$. Let $\vec{a}^{\prime}$ be the tuple whose $i$ th component $a_{i}^{\prime}$ is $1 / 2$ if $b_{i}=0$, and $b_{i}$ otherwise. We define the dipath $\beta$ by $\left.\beta(t)=\left[c_{k+1},(1-t) \vec{b}+t \vec{a}^{\prime}\right)\right], t \in[0,1]$. Note that $\beta$ is indeed monotonic, because $b_{i} \leq a_{i}^{\prime}$ for every $i$. One easily checks that $\beta(0)=\pi(1)$, and that the carrier sequence of $\langle\beta\rangle$ is $c_{k} \preceq c_{k+1}$ : for $t=0$, $\mathcal{C}(\beta(0))=(\pi(1))=c_{k}$, and, for $\left.t \neq 0, \beta(t)=\left[c_{k+1},(1-t) \vec{b}+t \vec{a}^{\prime}\right)\right]$ where no component of $(1-t) \vec{b}+t \vec{a}^{\prime}$ is equal to 0 or 1 , so its carrier $\mathcal{C}(\beta(t))$ is $c_{k+1}$. It follows that $\mathcal{C}(\langle\pi \star \beta\rangle)=c_{0} \preceq c_{1} \preceq \cdots \preceq c_{k} \preceq c_{k+1}$.

- If $c_{k+1}$ is a future boundary of $c_{k}$, then $c_{k+1}$ is of the form $\partial_{i_{p}}^{1} \ldots \partial_{i_{0}}^{1} c_{k}$ with $i_{0}>\ldots>i_{p}$, and $\pi(1)=\left[c_{k}, \vec{a}\right]$ for some tuple $\vec{a}$ whose components $a_{i}$ are all different from 0 or 1 (because $\mathcal{C}(\pi(1))=c_{k}$ ). Let $\vec{b}$ be the tuple obtained from $\vec{a}$ by changing the $i$ th component into 1 if and only if $i \in\left\{i_{0}, \cdots, i_{p}\right\}$. In other words, let $b_{i}=1$ if $i \in\left\{i_{0}, \cdots, i_{p}\right\}, b_{i}=a_{i}$ otherwise. One can therefore write $\vec{b}$ as $\delta_{i_{0}}^{1} \cdots \delta_{i_{p}}^{1} \vec{b}^{\prime}$, where $\vec{b}^{\prime}$ is the tuple obtained from $\vec{b}$ by removing its components of indices $i_{0}, \ldots, i_{p}$. Define the dipath $\beta$ by $\beta(t)=\left[c_{k},(1-t) \vec{a}+t \vec{b}\right]$. This is monotonic because $a_{i} \leq b_{i}$ for every $i$. For $t \neq 1$, no component of $(1-t) \vec{a}+t \vec{b}$ is equal to 0 or 1 , so $\mathcal{C}(\beta(t))=c_{k}$, and for $t=1, \beta(1)=\left[c_{k}, \vec{b}\right]=\left[c_{k+1}, \vec{b}^{\prime}\right]$, which shows that $\mathcal{C}(\beta(1))=c_{k+1}$ since no component of $\overrightarrow{b^{\prime}}$ is equal to 0 or 1 . Again, it follows that $\mathcal{C}(\langle\pi \star \beta\rangle)=c_{0} \preceq c_{1} \preceq \cdots \preceq c_{k} \preceq c_{k+1}$.

Proof. [C is the functorial part of an open map]

- $\mathcal{C}$ is surjective on objects : given a discrete trace $c_{0}, \ldots, c_{k}$ of $K, \mathcal{C}\left(\widehat{c_{0}, \ldots, c_{k}}\right)=$ $c_{0}, \ldots, c_{k}$

- $\mathcal{C}$ has the lifting property : let $(\alpha, \beta)$ be a morphism of $\mathcal{T}_{K}$ i.e. a pair of a discrete trace $\alpha$ from $x^{\prime}$ to $x$ and of a discrete trace $\beta$ from $y$ to $y^{\prime}$ such that there is a discrete trace from $x$ to $y$. Given a pair $(s, t)$ of points of $\overrightarrow{G e o m}(K)$ such that $\mathcal{C}(s)=x$ and $\mathcal{C}(t)=y$. Then using the previous lemma and an induction, we can find traces $\alpha^{\prime}$ from $s^{\prime}$ to $s$ and $\beta^{\prime}$ from $t$ to $t^{\prime}$ such that $\mathcal{C}\left(\alpha^{\prime}\right)=\alpha$ and $\mathcal{C}\left(\beta^{\prime}\right)=\beta$, which is what is required.

We now need to build a natural isomorphism

$$
\sigma: \vec{H}_{n}(\overrightarrow{\operatorname{Geom}}(K)) \longrightarrow \vec{h}_{n}(K) \circ \mathcal{C}
$$

In other words, we need to build for every $a, b$ in $\overrightarrow{G e o m}(K)$ such that there is a dipath from $a$ to $b$ a group isomorphism

$$
\sigma_{a, b}: \vec{H}_{n}(\overrightarrow{\operatorname{Geom}}(K))(a, b) \longrightarrow \vec{h}_{n}(K)(\mathcal{C}(a), \mathcal{C}(b))
$$


that is natural, in the sense that, for every extension $(\langle\alpha\rangle,\langle\beta\rangle)$ from $(a, b)$ to $\left(a^{\prime}, b^{\prime}\right)$, the following square commutes:

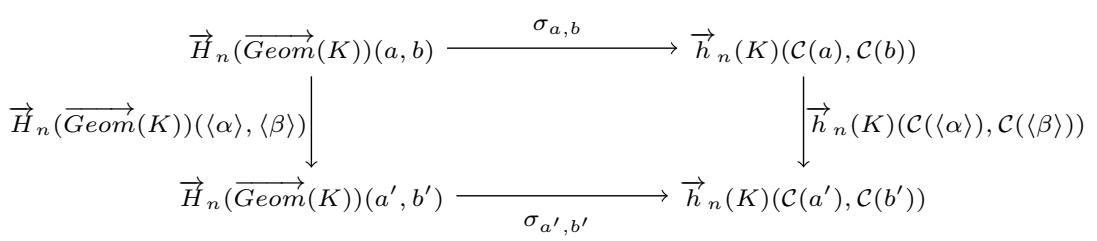

Let $x$ and $y$ such that there is a dipath from $x$ to $y$. Every cube $I^{k}$ has a lattice structure whose meet $\wedge$ is pointwise min and whose join $\vee$ is pointwise max. Write $x$ as $[\mathcal{C}(x), \vec{a}]$, and let $x_{-}=[\mathcal{C}(x), \vec{a} \wedge \bullet]$. Recall that $\bullet=\left(\frac{1}{2}, \cdots, \frac{1}{2}\right)$, and that $\widehat{\mathcal{C}(x)}=[\mathcal{C}(x), \bullet]$. Similarly, let $\widehat{\mathcal{C}(y)}=[\mathcal{C}(y), \bullet]$, and we define $y_{+}=$ $[\mathcal{C}(y), \vec{b} \vee \bullet]$, where $y=[\mathcal{C}(y), \vec{b}]$. The situation is illustrated here :
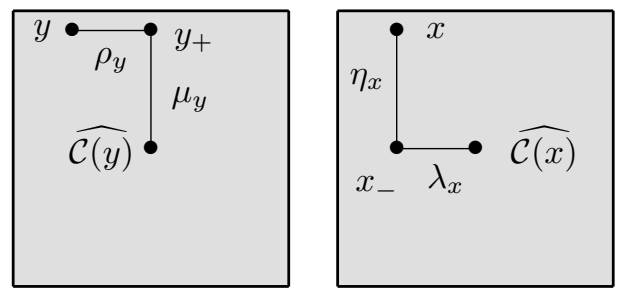

There are obvious dipaths $\eta_{x}, \lambda_{x}, \mu_{y}, \rho_{y}$ as displayed there, too. Let us make formal the construction of the dipath $\eta_{x}$. The other three are similar. This is a dipath from $x_{-}=[\mathcal{C}(x), \vec{a} \wedge \bullet]$ to $x=[\mathcal{C}(x), \vec{a}]$, and so we just let $\eta_{x}(t)=[\mathcal{C}(x),(1-t)(\vec{a} \wedge \bullet)+t \vec{a}]$. Those dipaths induce continuous maps between trace spaces by concatenation. For example, there is a continuous map $\eta_{x}^{*}$ : $\overrightarrow{\mathfrak{T}}(\overrightarrow{\operatorname{Geom}}(K))(x, y) \longrightarrow \overrightarrow{\mathfrak{T}}(\overrightarrow{G e o m}(K))\left(x_{-}, y\right)$ that sends each trace $\langle\pi\rangle$ to $\left\langle\eta_{x}\right.$ * $\pi\rangle$. Similarly, for the other three.

Lemma 3.9 The map $\eta_{x}^{*}$ is a homotopy equivalence.

Proof. By abuse of language, write $\eta_{x}^{*}(\pi)$ for the dipath $\eta_{x} \star \pi$ as wellwe reason on spaces of dipaths first, then take a reparametrization quotient. Accordingly, let $P(X ; x, y)$ denote the space of dipaths from $x$ to $y$ in $X=$ $\overrightarrow{\operatorname{Geom}}(K)$, with the usual compact-open topology. (The space $\overrightarrow{\mathfrak{T}}(X)(x, y)$ is a quotient of this space.)

Observe that $\eta_{x}^{*}$ maps $P(X ; x, y)$ to $P\left(X ; x_{-}, y\right)$. We need to build a map $\nu: P\left(X ; x_{-}, y\right) \longrightarrow P(X ; x, y)$ such that $\eta_{x}^{*} \circ \nu$ and $\nu \circ \eta_{x}^{*}$ are homotopic to the identity.

For every dipath $\pi$ from $x$ to $y$, the carrier sequence $c_{0}, c_{1}, \cdots, c_{k}$ of $\eta_{x}^{*}(\pi)$ is equal to that of $\pi$. In the other direction, we shall define $\nu$ so that it also preserves the carrier sequence. This will turn out to be the crucial property that will allow us to conclude. 
For every dipath $\pi$ from $x_{-}$to $y$, with carrier sequence $c_{0}, c_{1}, \cdots, c_{k}$, and with times of change $0=t_{0} \leq t_{1} \leq \cdots \leq t_{k} \leq t_{k+1}=1$, we define $\nu(\pi)$ as follows. We abuse the notation $\vee$, and write $[c, \vec{a}] \vee_{c}[c, \vec{b}]$ for $[c, \vec{a} \vee \vec{b}]$. The three occurrences of $c$ must be the same for this notation to make sense, but our intuition is best served by ignoring the $c$ subscript to $\mathrm{V}$, and to understand this as taking maxes, componentwise, in a local cube $c$. We then define $\nu(\pi)(u)$ for increasing values of $u$, inductively, as $x \vee_{c_{0}} \pi(u)$ for $u \in\left[t_{0}, t_{1}\right]$, as $\nu(\pi)\left(t_{1}\right) \vee_{c_{1}}$ $\pi(u)$ for $u \in\left[t_{1}, t_{2}\right], \ldots$, and finally as $\nu(\pi)\left(t_{k}\right) \vee_{c_{k}} \pi(u)$ for $u \in\left[t_{k}, t_{k+1}\right]$.

On $\left[t_{0}, t_{1}\right], \nu(\pi)$ is a continuous monotonic map, with value $\nu(\pi)(0)=x \vee_{c_{0}}$ $x_{-}=x$ at $u=t_{0}=0$, and with value $\nu(\pi)\left(t_{1}\right)=x \vee_{c_{0}} \pi\left(t_{1}\right)$ at $u=t_{1}$.

Let us show by induction on $j$ that for every $u$ with $0 \leq u \leq t_{j}, \mathcal{C}(\nu(\pi)(u))=$ $\mathcal{C}(\pi(u))$. For $j=0$, this says that $\mathcal{C}(x)=\mathcal{C}\left(x_{-}\right)$, which is by construction of $x_{-}$. Otherwise, by induction hypothesis, for every $u$ with $0 \leq u \leq t_{j}$, $\mathcal{C}(\nu(\pi)(u))=\mathcal{C}(\pi(u))$. Let $t_{j}<u \leq t_{j+1}$. We can write $\pi\left(t_{j}\right)$ as $\left[c_{j},\left(b_{1}, \ldots, b_{m}\right)\right]$ and $\pi(u)$ as $\left[c_{j},\left(a_{1}, \ldots, a_{m}\right)\right]$, where $b_{i} \leq a_{i}$ for every $i$.

- If $u<t_{j+1}$, by the properties of the carrier sequence, $\mathcal{C}(\pi(u))=c_{j}$, so with $0<a_{i}<1$ for every $i$. Since $b_{i} \leq a_{i}, b_{i}<1$ for every $i$. Let us write $\nu(\pi)\left(t_{j}\right)$ as $\left[c_{j},\left(b_{1}^{\prime}, \ldots, b_{m}^{\prime}\right)\right]$. Since $\mathcal{C}\left(\nu(\pi)\left(t_{j}\right)\right)=\mathcal{C}\left(\pi\left(t_{j}\right)\right), b_{i}=1$ iff $b_{i}^{\prime}=1$. It follows that $b_{i}^{\prime}<1$ for every $i$. Therefore $0<\max \left(a_{i}, b_{i}^{\prime}\right)<1$, so $\mathcal{C}(\nu(\pi)(u))=c_{k}$.

- If $u=t_{j+1}$, we observe that $\max \left(a_{i}, b_{i}^{\prime}\right)$ is equal to 1 , resp. to 0 , resp. in $(0,1)$, if and only if $a_{i}$ is. This observation is enough to conclude that $\mathcal{C}\left(\nu(\pi)\left(t_{j+1}\right)\right)=\mathcal{C}\left(\pi\left(t_{j+1}\right)\right)$, and is proved as follows. If $a_{i}=1$, then $\max \left(a_{i}, b_{i}^{\prime}\right)=1$. If $a_{i}=0$ then $b_{i}=0$; moreover, since $\mathcal{C}\left(\nu(\pi)\left(t_{j}\right)\right)=$ $\mathcal{C}\left(\pi\left(t_{j}\right)\right), b_{i}=0$ iff $b_{i}^{\prime}=0$, so $b_{i}^{\prime}=0$, from which we obtain $\max \left(a_{i}, b_{i}^{\prime}\right)=0$. Finally, if $0<a_{i}<1$ then $b_{i}<1$, and $b_{i}^{\prime}<1\left(\right.$ since $\mathcal{C}\left(\nu(\pi)\left(t_{j}\right)\right)=\mathcal{C}\left(\pi\left(t_{j}\right)\right)$, $b_{i}=1$ iff $\left.b_{i}^{\prime}=1\right)$, so $0<\max \left(a_{i}, b_{i}^{\prime}\right)<1$.

This finishes our argument that $c_{0}, \ldots, c_{k}$ is the carrier sequence of $\nu(\pi)$, with times of change $0=t_{0} \leq \ldots \leq t_{k+1}=1$.

It remains to show that $\nu(\pi)(1)=y$. This is the only place where we need the $\epsilon$ mapping. The above argument works in general precubical sets, not just cubical complexes. On the contrary, we need the specific features of cubical complexes to show that $\nu(\pi)(1)=y$.

We know that $\mathcal{C}(y)=\mathcal{C}(\nu(\pi)(1))=c_{k}$. Moreover, $y$ is below $\nu(\pi)(1)$ in the ordering $\leq$ of the pospace $\overrightarrow{G e o m}(K)$, because $\nu(\pi)(1)=\nu(\pi)\left(t_{k}\right) \vee_{c_{k}} \pi(1)=$ $\nu(\pi)\left(t_{k}\right) \vee_{c_{k}} y$. Suppose that $\nu(\pi)(1) \not \leq y$. Because $K$ is a cubical complex, we can make use of the $\epsilon$ isomorphism. From $\nu(\pi)(1) \not \leq y$, we obtain $\epsilon(\nu(\pi)(1)) \not{Z}$ $\epsilon(y)$. Let us write $\epsilon\left(\nu(\pi)\left(t_{j}\right)\right)$ as $\left(x_{1}^{j}, \ldots, x_{d}^{j}\right)$ and $\epsilon\left(\pi\left(t_{j}\right)\right)$ as $\left(y_{1}^{j}, \ldots, y_{d}^{j}\right)$. We show that $\epsilon\left(\nu(\pi)\left(t_{j}\right)\right) \not \leq \epsilon(y)$ by decreasing induction on $j$. The case $j=$ $k+1$ is by assumption. Suppose $\epsilon\left(\nu(\pi)\left(t_{j+1}\right)\right) \not \leq \epsilon(t)$. There must be an index $m \in\{1,2, \cdots, d\}$ such that $x_{m}^{j+1}>y_{m}^{k+1}$. It is easy to see that the identity $\epsilon\left([c, \vec{a}] \vee_{c}[c, \vec{b}]\right)=\epsilon([c, \vec{a}]) \vee \epsilon([c, \vec{b}])$ holds, where the right-hand $\vee$ is componentwise max in $\mathbb{R}^{d}$ (a property that is not usually implied by the mere fact that $\epsilon$ is an isomorphism). From that and $\nu(\pi)\left(t_{j+1}\right)=\nu(\pi)\left(t_{j}\right) \vee_{c_{j}} \pi\left(t_{j+1}\right)$, 
we infer that $x_{m}^{j+1}=\max \left(x_{m}^{j}, y_{m}^{j+1}\right)$, hence $y_{m}^{j+1} \leq x_{m}^{j+1}$. But $\pi$ restricts to a dipath from $t_{j}$ to $y$, so $\epsilon\left(\pi\left(t_{j}\right)\right) \leq \epsilon(t)$, and therefore $y_{m}^{j+1} \leq y_{m}^{k+1}<x_{m}^{j+1}$. From $y_{m}^{j+1}<x_{m}^{j+1}$ and $x_{m}^{j+1}=\max \left(x_{m}^{j}, y_{m}^{j+1}\right)$, we obtain $x_{m}^{j+1}=x_{m}^{j}$, whence $x_{m}^{j}>y_{m}^{k+1}$. In particular, $\epsilon\left(\nu(\pi)\left(t_{j}\right)\right) \not \leq \epsilon(y)$.

Taking $j=0$, this implies that $\epsilon(x) \not \leq \epsilon(y)$. This is impossible, since there is a dipath from $x$ to $y$.

We have constructed a map $\nu$ such that $\pi$ and $\nu(\pi)$ have same carrier sequence. We can now conclude by the following lemma:

Lemma 3.10 Let $F, G: P(X ; s, t) \longrightarrow P\left(X ; s^{\prime}, t^{\prime}\right)$ such that:

- for every pair of dipaths $p, q$ that are equivalent modulo reparametrization, $F(p)$ and $F(q)$ are equivalent modulo reparametrization-so $F$ induces $\tilde{F}: \overrightarrow{\mathfrak{T}}(X)(s, t) \longrightarrow \overrightarrow{\mathfrak{T}}(X)\left(s^{\prime}, t^{\prime}\right)$, and similarly for $G$.

- for every $\pi, F(\pi)$ and $G(\pi)$ have the same carrier sequence.

Then $\tilde{F}$ and $\tilde{G}$ are homotopic.

Proof. Let $C\left(X ; s^{\prime}, t^{\prime}\right)$ is the subspace of $P\left(X ; s^{\prime}, t^{\prime}\right) \times P\left(X ; s^{\prime}, t^{\prime}\right)$ that consists of pairs of dipaths that have the same carrier sequence. The key ingredient consists in constructing a continuous map $\Gamma: I \times C\left(X ; s^{\prime}, t^{\prime}\right) \longrightarrow P\left(X ; s^{\prime}, t^{\prime}\right)$ in such a way that $\Gamma(0,(p, q))=p$ and $\Gamma(1,(p, q))=q$. Let $c_{0}, c_{1}, \cdots, c_{k}$ be the common carrier sequence to $p$ and $q$, let $t_{0} \leq t_{1} \leq \cdots \leq t_{k+1}$ be the times of change for $p$, and $s_{0} \leq s_{1} \leq \cdots \leq s_{k+1}$ be the times of change for $q$. Define $u_{i}(t)=t s_{i}+(1-t) t_{i}$ for $t \in[0,1], 0 \leq i \leq k+1$. For every $u \in\left[u_{i}(t), u_{i+1}(t)\right]$, define $v$ as $\frac{u-u_{i}(t)}{u_{i+1}(t)-u_{i}(t)}$. (This is defined provided $u_{i}(t) \neq u_{i+1}(t)$; if this is not the case, let $v=0$.) Then $p\left(v\left(t_{i+1}-t_{i}\right)+t_{i}\right)$ is of the form $\left[c_{i},\left(a_{1}^{u}, \ldots, a_{m}^{u}\right)\right]$ and $q\left(v\left(s_{i+1}-s_{i}\right)+s_{i}\right)$ is of the form $\left[c_{i},\left(b_{1}^{u}, \ldots, b_{m}^{u}\right)\right]$. We then define $\Gamma(t, p, q)(u)=\left[c_{i},(1-t) a_{j}^{u}+t b_{j}^{u}\right]$.

We have to define a homotopy $H: I \times \overrightarrow{\mathfrak{T}}(X)(s, t) \longrightarrow \overrightarrow{\mathfrak{T}}(X)\left(s^{\prime}, t^{\prime}\right)$. It will be defined as the composition of:

- $i d \times \kappa: I \times \overrightarrow{\mathfrak{T}}(X)(s, t) \longrightarrow I \times P(X ; s, t)$, where $\kappa$ is a continuous map from $\overrightarrow{\mathfrak{T}}(X)(s, t)$ to $P(X ; s, t)$, defined in such a way that $\langle\kappa(\langle\pi\rangle)\rangle=\langle\pi\rangle$ for every trace $\langle\pi\rangle$, therefore defining a canonical dipath representing a given trace. The existence of such a map is shown by Raussen in [43], as the composition norm $\circ \vec{s}$ of two more elementary maps.

- $i d \times(F, G): I \times P(X ; s, t) \longrightarrow I \times C\left(X ; s^{\prime}, t^{\prime}\right)$, where $(F, G)$ maps $\pi$ to $(F(\pi), G(\pi))$.

- $\Gamma: I \times C\left(X ; s^{\prime}, t^{\prime}\right) \longrightarrow P\left(X ; s^{\prime}, t^{\prime}\right)$, as defined above.

- and $\left\langle_{-}\right\rangle: P\left(X ; s^{\prime}, t^{\prime}\right) \longrightarrow \overrightarrow{\mathfrak{T}}(X)\left(s^{\prime}, t^{\prime}\right)$, which maps each dipath to its trace. 
We compute: $H(0,\langle\pi\rangle)=\langle\Gamma(0,(F(\kappa(\langle\pi\rangle)), G(\kappa(\langle\pi\rangle)))\rangle=\langle F(\kappa(\langle\pi\rangle))\rangle=\tilde{F}(\langle\pi\rangle)$. Similarly, $H\left(1,{ }_{-}\right)=\tilde{G}$ and therefore $H$ is an homotopy from $\tilde{F}$ to $\tilde{G}$.

It only remains to prove that the construction is natural. The following diagram:

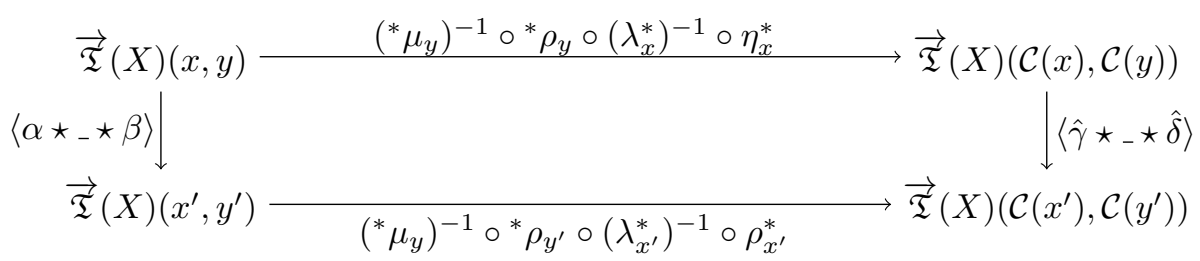

where $\gamma=\mathcal{C}(\alpha)$ and $\delta=\mathcal{C}(\beta)$ is commutative modulo homotopy because of the previous lemma and so the same diagram is commutative in homology, which proves the naturality.

Corollary 3.11 Let $K$ be a cubical complex, and $K^{\prime}$ be a subdivision of $K$. Then $\vec{h}_{n}(K)$, and $\vec{h}_{n}\left(K^{\prime}\right)$ are bisimilar.

Proof. $\quad K$ and $K^{\prime}$ have isomorphic (in the category of pospaces) geometric realization, then by functoriality of natural homology, they have isomorphic (then bisimilar) natural homology then their discrete natural homology are bisimilar.

\subsection{Hochschild-Mitchell homology theories and natural sys- tems}

Instead of taking as a base space the category of factorization of a trace category, i.e. the category $\mathcal{T}_{X}$ of Definition 2.5, we can take the "smaller" category of pairs of base points (initial, final, see Definition 3.12), together with extensions (in the past, in the future). We will see that the homological information we can construct on this base space is akin to a Hochschild-Mitchell homology theory $[38,39]$ and that the comparison functor from Baues-Wirshing homology (our homological diagrams, i.e. natural systems of Definition 2.9) actually induces an equivalence under bisimilarity.

Definition 3.12 We define $\preceq$ the preorder on $X$ defined as $x \preceq y$ iff there exists a dipath from $x$ to $y$. We define the category $\mathcal{P}_{X}$ whose:

- objects are pairs of points $(x, y)$ of $X$ such that $x \preceq y$

- morphisms (called extensions) from $(x, y)$ to $\left(x^{\prime}, y^{\prime}\right)$ are pairs $(\langle\alpha\rangle,\langle\beta\rangle)$ of trace of $X$ with $\alpha$ dipath from $x^{\prime}$ to $x$ and $\beta$ dipath from $y$ to $y^{\prime}$

We have a functor $\kappa_{X}: \mathcal{T}_{X} \longrightarrow \mathcal{P}_{X}$ which maps each trace to its end points. 
Definition 3.13 We define the trace diagram as the functor $\vec{T}(X): \mathcal{P}_{X} \longrightarrow$ Top which maps :

- every pair $(x, y)$ with $x \preceq y$ to $\overrightarrow{\mathfrak{T}}(X)(x, y)$

- every extension $(\langle\alpha\rangle,\langle\beta\rangle)$ from $(x, y)$ to $\left(x^{\prime}, y^{\prime}\right)$ to the continuous function $\left\langle\alpha \star_{-} \star \beta\right\rangle: \overrightarrow{\mathfrak{T}}(X)(x, y) \longrightarrow \overrightarrow{\mathfrak{T}}(X)\left(x^{\prime}, y^{\prime}\right) \quad\langle p\rangle \mapsto\langle\alpha \star p \star \beta\rangle$

As in definition 2.9, we can define a diagram of abelian groups :

Definition 3.14 We define for $n \geq 1, \vec{H}_{n}^{H M}(X): \mathcal{P}_{X} \longrightarrow \boldsymbol{A b}$ composing $\vec{T}(X)$ with the functor which maps its $n-1$ th homology group. In particular, $\vec{H}_{n}(X)=\vec{H}_{n}^{H M}(X) \circ \kappa_{X}$.

As in pospaces, we can define the category $\mathcal{P}_{K}$ whose objects are pairs $(x, y)$ of elements of $K$ such that there exists a discrete trace from $x$ to $y$ and morphisms from $(x, y)$ to $\left(x^{\prime}, y^{\prime}\right)$ are pairs $(\alpha, \beta)$ of a discrete trace $\alpha$ from $x^{\prime}$ to $x$ and of a discrete trace $\beta$ from $y$ to $y^{\prime}$. We have a functor $\kappa_{K}: \mathcal{T}_{K} \longrightarrow \mathcal{P}_{K}$ which maps each discrete trace to its end points. Then, when $K$ is a cubical complex, previous remarks define a functor $\imath^{\wedge M}: \mathcal{P}_{K} \longrightarrow \mathcal{P}_{\overrightarrow{\operatorname{Geom}(K)}}$. As previously, we can then define a notion of natural homology of a cubical complex :

Definition 3.15 Let $K$ be a cubical complex. For $n \geq 1$, we define the $n$-th natural homology of $K$ or discrete natural homology $\vec{h}_{n}^{H}{ }^{M}(K): \mathcal{T}_{K}^{d} \longrightarrow \boldsymbol{A b}$ as

$$
\vec{h}_{n}^{H M}(K)=\vec{H}_{n}^{H M}(\overrightarrow{\operatorname{Geom}}(K)) \stackrel{\curvearrowright}{H M}^{H M}
$$

In particular, $\vec{h}_{n}(X)=\vec{h}_{n}^{H M}(X) \circ \kappa_{X}$.

All those definitions coincide up-to bisimulation :

\section{Proposition 3.16}

- for every pospace $X,\left(\kappa_{X}, 1\right): \vec{H}_{n}^{H M}(X) \longrightarrow \vec{H}_{n}(X)$ is an open map

- for every cubical complex $K,\left(\kappa_{K}, 1\right): \vec{H}_{n}^{H}{ }^{M}(K) \longrightarrow \vec{H}_{n}(K)$ is an open map

- for every cubical complex $K$, like in Theorem 3.6, there is an open map $(\mathcal{C}, \sigma): \vec{H}_{n}^{H M}(\overrightarrow{\operatorname{Geom}}(K)) \longrightarrow \vec{h}_{n}^{H M}(K)$

\section{Diagrammatic properties of natural homology}

We are now in a position to study the diagrammatic properties of our natural homology. By diagrammatic properties, we mean Eilenberg-Steenrod [5] axioms for homology theories, and in particular, exactness properties. We first begin by classical Hurewicz theorems in Section 4.1 and then proceed by looking at 
the dimension, homotopy and additivity axioms. Finally, we define and study exactness of diagrams in natural systems, in Sections 4.2.2, 4.2.3 and 4.2.4. Many properties of classical homology hold true, but not quite the exactness axiom of Eilenberg-Steenrod. Looking for some form of an excision axiom is also very intricate for natural homology, and is left for future work.

\subsection{Hurewicz theorems}

\section{Definition 4.1}

We say that $X$ is 1-connected if for every trace $\langle p\rangle, \vec{\Pi}_{1}(X)(\langle p\rangle)$ is a singleton. We say that $X$ is $n$-connected with $n \geq 2$ if it is $n-1$-connected and if for every trace $\langle p\rangle, \vec{\Pi}_{n}(X)(\langle p\rangle)$ is a trivial group.

Now, we have for free a Hurewitz theorem between natural homotopy and natural homology :

\section{Theorem 4.2}

Free $\circ \vec{\Pi}_{1}(X)$ is isomorphic in Diag $(\boldsymbol{A} \boldsymbol{b})$ to $\vec{H}_{1}(X)$ where Free : Set $\longrightarrow \boldsymbol{A} \boldsymbol{b}$ is the functor which gives the free group.

If $X$ is 1-connected, Ab० $\vec{\Pi}_{2}(X)$ is isomorphic in Diag $(\boldsymbol{A} \boldsymbol{b})$ to $\vec{H}_{2}(X)$ where $A b: \boldsymbol{G r p} \longrightarrow \boldsymbol{A} \boldsymbol{b}$ is the functor which gives the abelianization.

If $X$ is $n-1$ connected with $n \geq 3, \vec{\Pi}_{n}(X)$ is isomorphic in Diag $(\boldsymbol{A b})$ to $\vec{H}_{n}(X)$.

Proof.

This is just a consequence of the naturality in the classical Hurewicz theorem. below :

We can refine this result to our Hochschild-Mitchell [38, 39] form of homology

Theorem 4.3 Free $\circ \vec{\Pi}_{1}(X)$ is bisimilar to $\vec{H}_{1}^{H M}(X)$ where Free : Set $\longrightarrow$ $\boldsymbol{A} \boldsymbol{b}$ is the functor which gives the free group.

If $X$ is 1-connected, Ab० $\vec{\Pi}_{2}(X)$ is bisimilar to $\vec{H}_{2}^{H M}(X)$ where $A b:$ Grp $\longrightarrow$ $\boldsymbol{A} \boldsymbol{b}$ is the functor which gives the abelianization.

If $X$ is $n-1$ connected with $n \geq 3, \vec{\Pi}_{n}(X)$ is bisimilar to $\vec{H}_{n}^{H M}(X)$.

Proof. Consequence of theorem 4.2 and proposition 3.16.

\subsection{The homological category of diagrams}

Another important ingredient of classical homology theories is that they all satisfy the Eilenberg-Steenrod axioms [5].

We first examine the simpler axioms : the dimension, homotopy and additivity axioms. The dimension axiom, in abelian homology theories, states that the homology $H_{n}$ of a point is the object zero for all $n>0$. The homotopy axiom states that a weak homotopy equivalence induces an isomorphism in homology. Finally, the additivity axiom states that the homology of a coproduct 
is the coproduct of the homologies. We will see in Section 4.2.1 that all three are trivial in our setting, if of course we interpret everything up to bisimulation.

The exactness axiom states that the so-called relative homology long sequence is exact. We will first ask ourselves whether natural homology functors transform short exact sequences (as e.g. the ones defining quotients and relative pairs of spaces) into long exact sequences, as standard homology functors do, as this is particularly useful for making actual homological calculations.

Exactness of diagrams as well as exact functors are generally defined in abelian categories [34]. Unfortunately, $\operatorname{Diag}(\mathbf{A b})$ is not abelian : for example, it does not have a zero object.

In order to be able to consider some sort of exactness property, we will consider the theory of homology in a non-abelian setting of [16, 17]. In Section 4.2.2 we recap the notions of semi-exact and exact categories from Grandis' work, and show that natural homology lives in a semi-exact category $(\operatorname{Diag}(\mathbf{A b}))$. This allows for defining exact sequences and exact functors, as well as do a certain amount of diagram chasing. Then in Section 4.2.3, we recap the notion of a homological category, in which we can do more, we can define quotients, hence homology functors, abstractly, and still carry on diagram chasing. We show that $\operatorname{Diag}(\mathbf{A b})$ is homological. In Section 4.2.4 we go one step further and introduce modular categories. Homological categories only map short exact sequences in a sequence of order 2, whereas modular categories map them onto long exact sequences. We show that unfortunately, $\operatorname{Diag}(\mathbf{A b})$ is not modular. Still, we can prove that the relative homology long sequence is exact, in Section 4.2.5.

\subsubsection{Dimension, homotopy and additivity axioms}

We first have, trivially :

Proposition 4.4 (Dimension axiom) The nth natural homology (for $n \neq 0$ ) of a point is trivial, i.e. it is the functor from the category with one object and one morphism which maps this object to the trivial group.

Then the homotopy axiom is easy to prove, with natural homology :

Proposition 4.5 (Homotopy axiom) Let $f: X \longrightarrow Y$ be a dmap such that for all $n \leq 1, \vec{\Pi}_{n}(f): \vec{\Pi}_{n}(X) \longrightarrow \vec{\Pi}_{n}(Y)$ is on open map then for all $n \leq 1$, $\vec{H}_{n}(f): \overrightarrow{\vec{H}}_{n}(X) \longrightarrow \vec{H}_{n}(Y)$ is on open map.

Proof. The functorial part of $\vec{H}_{n}(f)$ is the same as of $\vec{\Pi}_{n}(f)$ and the isomorphism in homology comes from the homotopy axiom in singular homology.

This trivially implies that if $f$ induces a bisimulation equivalence between the homotopy functors for $X$ and $Y$, which is the natural weak-equivalence we have in our directed homotopy theory, then $f$ induces a bisimulation equivalence between the homology functors for $X$ and $Y$.

Finally, we consider the additivity axiom. First, we make explicit the coproduct construction in $\operatorname{Diag}(\mathcal{M})$ (although much more can be said about limits and colimits, see Appendix .1) : 
Lemma 4.6 (Coproducts in $\operatorname{Diag}(\mathcal{M})$ ) The coproducts of diagrams is the disjoint union i.e. let $\left(F_{i}: \mathcal{C}_{i} \longrightarrow \mathcal{M}\right)_{i \in I}$ be a family of diagrams then the its coproduct is $\coprod_{i \in I} F_{i}: \coprod_{i \in I} \mathcal{C}_{i} \longrightarrow \mathcal{M}$ which maps $c \in \mathcal{C}_{i}$ to $F_{i}(c)$.

Proposition 4.7 (Additivity axiom) If $X$ is the disjoint union of $X_{i}, i \in I$, then for all $n \in \mathbb{N}, \vec{H}_{n}(X)=\coprod_{i \in I} \vec{H}_{n}\left(X_{i}\right)$.

\subsubsection{Semi-exact categories}

We now want to be able to talk about exact sequences. Let $\mathcal{A}$, a category. An ideal of $\mathcal{A}$ is a set of morphisms stable under left and right compositions by any (composable) morphism of $\mathcal{A}$.

Let $N$, an ideal of $\mathcal{A}$. We call the morphisms in $N$, the null morphisms. A null object is an object of $\mathcal{A}$ whose identity is null.

We say that $N$ is closed if every null morphism factorises through a null object i.e. for every $f: A \rightarrow B \in N$, there exists a null object $C$ and two morphisms $g: A \rightarrow C$ and $h: C \rightarrow B$ such that $f=h \circ g$.

The kernel (with respect to $N$ ) of a morphism $f: A \rightarrow B$ of $\mathcal{A}$ is characterized (if it exists) up to isomorphism by the following property :

- ker $f: \operatorname{Ker} f \rightarrow A$ such that $f \circ \operatorname{ker} f \in N$

- for all $g: C \rightarrow A$ such that $f \circ g \in N$, there exists a unique $h: C \rightarrow \operatorname{ker} f$ such that $g=\operatorname{Ker} f \circ h$

We define dually, the cokernel.

A semi-exact category is a pair $(\mathcal{A}, N)$ where $N$ is a closed ideal of the category $\mathcal{A}$ such that every morphism of $\mathcal{A}$ has a kernel and a cokernel with respect to $N$.

We call normal mono (resp. normal epi), a morphism which is the kernel (resp. the cokernel) of a morphism.

We call image of a morphism $f, \operatorname{im} f=k e r$ cok $f$ and coimage, coim $f=$ cok ker $f$.

Definition 4.8 ([16]) The sequence

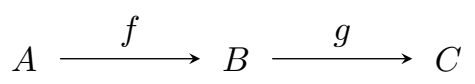

is said to be of order two (resp. short exact, exact) if $g \circ f$ is null (resp. if $f=\operatorname{ker} g$ and $g=\operatorname{cok} f$, if im $f=$ ker $g$ ).

Lemma 4.9 If $(\mathcal{M}, N)$ is semi-exact then $L=\left\{(\Phi, \tau) \mid \forall f, \tau_{f} \in N\right\}$ is a closed ideal of Diag $(\mathcal{M})$ and $\operatorname{Diag}(\mathcal{M})$ has kernels with respect to $L$.

Proof.

- $L$ is an ideal because $N$ is 
- $L$ closed : we know by 3.7 of [16] that in $\mathcal{M}, f: A \longrightarrow B$ is null iff $f$ factorizes through $\operatorname{ker} 1_{B}: \operatorname{Ker} 1_{B} \longrightarrow B$ and that $\operatorname{ker} 1_{B}$ is a null object. So, if $(\Phi, \sigma):(F: \mathcal{C} \longrightarrow \mathcal{M}) \longrightarrow(G: \mathcal{D} \longrightarrow \mathcal{M})$ is null then it factorizes through $0_{G}$ where $0_{G}: \mathcal{D} \longrightarrow \mathcal{M}$ with $0_{G}(d)=\operatorname{ker} 1_{G(d)}$ and $0_{G}(f): 0_{G}(d) \longrightarrow 0_{G}\left(d^{\prime}\right)$ the unique morphism which makes this square commutative :

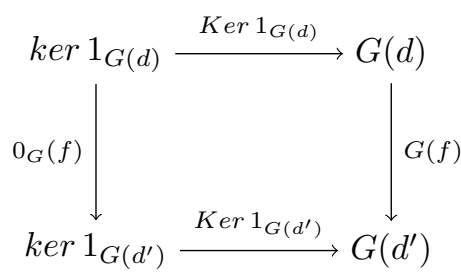

coming from the universal property of $\operatorname{Ker} 1_{G\left(d^{\prime}\right)}$.

- kernels : if $(\Phi, \sigma):(F: \mathcal{C} \longrightarrow \mathcal{M}) \longrightarrow(G: \mathcal{D} \longrightarrow \mathcal{M})$, we construct

$\operatorname{Ker}(\Phi, \sigma)=\left(\Phi_{\text {ker }}, \sigma_{\text {ker }}\right): \operatorname{ker}(\Phi, \sigma)=\left(F_{\text {ker }}: \mathcal{C}_{\text {ker }} \longrightarrow \mathcal{M}\right) \longrightarrow(F:$ $\mathcal{C} \longrightarrow \mathcal{M})$ as follows :

- $\mathcal{C}_{\text {ker }}=\mathcal{C}$

- $\Phi_{\text {ker }}=i d_{\mathcal{C}}$

- $F_{k e r}: \mathcal{C} \longrightarrow \mathcal{M}$ with $F_{k e r}(c)=k e r \sigma_{c}$ and $F_{k e r}(f)$ the unique morphism which makes the left square commutative :

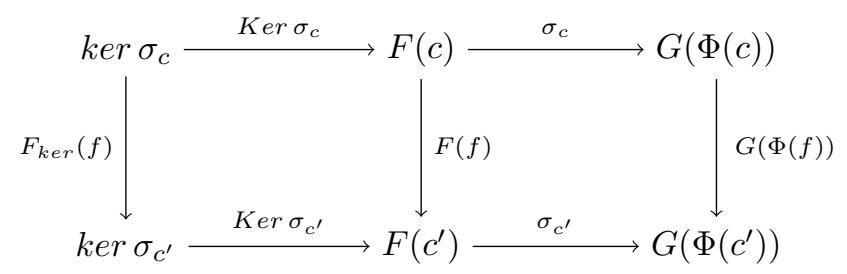

coming from the universal property of $\operatorname{Ker} \sigma_{c^{\prime}}$

- $\left(\sigma_{k e r}\right)_{c}=\operatorname{Ker} \sigma_{c}$

Corollary 4.10 Normal monos in $(\boldsymbol{D i a g}(\mathcal{M}), L)$ are the $(\Phi, \sigma)$ where $\Phi$ is an isofunctor and every $\sigma_{c}$ is a normal mono in $(\mathcal{M}, N)$.

Lemma 4.11 Diag $(\boldsymbol{A b})$ has cokernels with respect to $L$.

Proof. If $(\Phi, \sigma):(F: \mathcal{C} \longrightarrow \mathbf{A b}) \longrightarrow(G: \mathcal{D} \longrightarrow \mathbf{A b})$, we construct $\operatorname{Cok}(\Phi, \sigma)=\left(\Phi_{\text {cok }}, \sigma_{c o k}\right):(G: \mathcal{D} \longrightarrow \mathbf{A b}) \longrightarrow \operatorname{cok}(\Phi, \sigma)=\left(G_{c o k}: \mathcal{D}_{c o k} \longrightarrow\right.$ Ab) as follow :

- $\mathcal{D}_{c o k}=\mathcal{D}$ 
- $\Phi_{c o k}=i d_{\mathcal{D}}$

- Let $\Gamma=\left\{\left(R_{d}\right)_{d \in O b(\mathcal{D})} \mid R_{d}\right.$ subgroup of $G(d)$ containing all the elements of $\operatorname{Im} \sigma_{c}$ with $\Phi(c)=d$ and such that if $f: d \longrightarrow d^{\prime}$ then $\left.G(f)\left(R_{d}\right) \subseteq R_{d^{\prime}}\right\} . \quad \Gamma$ contains $(G(d))_{d \in O b(\mathcal{D})}$ and is stable under intersection. Define then $\left(H_{d}\right)_{d \in O b(\mathcal{D})}$ as the intersection of all the elements of $\Gamma$. Then $\left(H_{d}\right)_{d \in O b(\mathcal{D})} \in \Gamma$.

We also define $G_{c o k}: \mathcal{D} \longrightarrow$ Ab with $G_{c o k}(d)=G(d) / H_{d}$ and $G_{c o k}(f)([x])=$ $[G(f)(x)]$. This is well defined because $\left(H_{d}\right)_{d \in O b(\mathcal{D})} \in \Gamma$.

- $\left(\sigma_{c o k}\right)_{d}(x)=[x]$

You can observe that this is the coequalizer of $(\Phi, \sigma)$ and $(\Phi,(0: F(c) \longrightarrow$ $\left.G(\Phi(c)))_{c \in O b(\mathcal{C})}\right)$.

Corollary 4.12 Normal epis in $(\boldsymbol{D i a g}(\boldsymbol{A b}), L)$ are $(\Phi, \sigma)$ where $\Phi$ is an isofunctor and every $\sigma_{c}$ is surjective.

Theorem $4.13(\operatorname{Diag}(\boldsymbol{A b}), L)$ is semi-exact.

\subsubsection{Homological categories}

Now, to define homology, we have to be able to talk about sub-quotient as in the case of abelian groups i.e. if $K \subseteq H \subseteq G$ are abelian groups, we can define $H / K$. This is not the case in general groups even if $H$ and $K$ are normal sub-groups of $G$.

Definition 4.14 ([16]) We say that a morphism is exact if it factories as $n \circ q$ with $q$, a normal epi and $n$, a normal mono.

A semi-exact category $(\mathcal{A}, N)$ is said to be homological if :

- normal monos and normal epis are stable under composition

- if $m: B \rightarrow A$ is a normal mono and $q: A \rightarrow C$ is a normal epi with $m \geq \operatorname{ker} q$ in $\operatorname{Sub}(A)$ (i.e. there exists $k$, which is unique and monic, such that ker $q=m \circ k$ ) then $q \circ m$ is exact.

If $m: M \rightarrow A$ and $n: N \rightarrow A$ are two normal monos with $m \geq n$, and if $q=\operatorname{cok} n$, the object coim $q \circ m$ (isomorph to im $q \circ m$ ) defined up to isomorphism will be called $a$ sub-quotient of $A$ induced by $m \geq n$ and written $M / N$.

Proposition 4.15 $\operatorname{Diag}(A b)$ is homological.

\section{Proof.}

- the normal monos (resp. epis) are stable under composition by Corollaries 4.10 and 4.12 . 
- let $(\Phi, \sigma):(F: \mathcal{C} \longrightarrow \mathbf{A b}) \longrightarrow(G: \mathcal{D} \longrightarrow \mathbf{A b})$ be a normal mono. Thus $\Phi$ is an isofunctor. As normal mono/epi are stable under composition by an isomorphism, we can suppose wlog that $\Phi=i d_{\mathcal{C}}$ and $\mathcal{D}=\mathcal{C}$. The same way we can take $(\Psi, \tau):(G: \mathcal{C} \longrightarrow \mathbf{A b}) \longrightarrow(H: \mathcal{E} \longrightarrow \mathbf{A b})$ a normal epi with $\Psi=i d_{\mathcal{C}}$ and $\mathcal{C}=\mathcal{E}$. Then $(\Psi, \tau) \circ(\Phi, \sigma)=\left(i d_{\mathcal{C}},\left(\tau_{c} \circ \sigma_{c}\right)_{c \in O b(\mathcal{C})}\right)$. But in $\mathbf{A b}, \tau_{c} \circ \sigma_{c}=\iota_{c} \circ \eta_{c}$ where $\eta_{c}=\tau_{c} \circ \sigma_{c}: F(c) \longrightarrow \operatorname{Im} \tau_{c} \circ \sigma_{c}$ which is surjective and $\iota_{c}: \operatorname{Im} \tau_{c} \circ \sigma_{c} \longrightarrow H(c)$ the inclusion, which is injective. Moreover, $\left(\eta_{c}\right)_{c \in O b(\mathcal{C})}$ and $\left(\iota_{c}\right)_{c \in O b(\mathcal{C})}$ are natural and so $\left(i d_{\mathcal{C}}, \eta\right)$ is a normal epi, $\left(i d_{\mathcal{C}}, \iota\right)$ is a normal mono and $(\Psi, \tau) \circ(\Phi, \sigma)=\left(i d_{\mathcal{C}}, \iota\right) \circ$ $\left(i d_{\mathcal{C}}, \eta\right)$.

Definition $4.16([\mathbf{1 7}])$ Chain complexes in a semi-exact category $\mathcal{M}$ are defined the same way as in abelian groups requiring that the sequence of morphisms $C_{n+1} \stackrel{\partial_{n+1}}{\longrightarrow} C_{n} \stackrel{\partial_{n}}{\longrightarrow} C_{n-1}$ be of order two. A morphism of chain complexes $\left(f_{n}\right)_{n}:\left(C_{n}, \partial_{n}\right) \longrightarrow\left(C_{n}^{\prime}, \partial_{n}^{\prime}\right)$ is the data of morphisms $f_{n}: C_{n} \longrightarrow C_{n}^{\prime}$ such that $\partial_{n}^{\prime} \circ f_{n+1}=f_{n} \circ \partial_{n}$. We define this way a semi-exact category $C \bullet(\mathcal{M})$ which is homological when $\mathcal{M}$ is.

Then, we can define for a chain complex $A_{\bullet}=\left(A_{n}, \partial_{n}\right)$ :

- $Z_{n}\left(A_{\bullet}\right)=\operatorname{ker} \partial_{n-1}$

- $B_{n}\left(A_{\bullet}\right)=i m \partial_{n}$

- $H_{n}\left(A_{\bullet}\right)=Z_{n}\left(A_{\bullet}\right) / B_{n}\left(A_{\bullet}\right)$

and those constructions are functorial.

Definition 4.17 We define $\vec{C}:$ PoTop $\longrightarrow C_{\bullet}(\boldsymbol{D i a g}(\boldsymbol{A b}))$ composing $\vec{T}$ with the functor which maps a topological space to its singular chain complex. We can define its homology as in Section 2.2.

Proposition 4.18 For every $n \leq 0, H_{n} \circ \vec{C}=\vec{H}_{n+1}$.

Proof. A chain complex of diagram is the same as a diagram of chain complexes.

\subsubsection{Modularity and long exact sequences in homology}

In the theory of Grandis, we have the following theorem :

Theorem 4.19 ([17]) Let $\mathcal{M}$ a homological category. For each short exact sequence in $C_{\bullet}(\mathcal{M})$ :

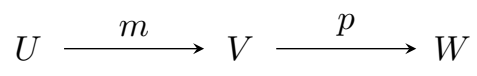

there exists a sequence of order two in $\mathcal{M}$ : 


$$
\cdots \longrightarrow H_{n}(V) \stackrel{H_{n}(p)}{\longrightarrow} H_{n}(W) \stackrel{\partial_{n}}{\longrightarrow} H_{n-1}(U) \stackrel{H_{n-1}(m)}{\longrightarrow} H_{n-1}(V) \longrightarrow \cdots
$$

which is natural in the short exact sequence.

Moreover, [17] gives some conditions for this sequence to be exact. In particular, those conditions are always satisfied iff $\mathcal{M}$ is modular.

A normal subobject of $F: \mathcal{C} \longrightarrow \mathbf{A b} \in \operatorname{Diag}(\mathbf{A b})$ is a morphism of the form $\left(i d_{\mathcal{C}}, \sigma\right)$ where every $\sigma_{c}$ is an inclusion into $F(c)$. The set of all normal subobjects of $F$ is a lattice whose order is inclusion, meet is intersection, join is union, $\perp$ is $\sigma_{c}=0$ and $T$ is $i d_{F(c)}$. Moreover, it is a modular lattice that is if $X \leq B$ then $X \vee(A \wedge B)=(X \vee A) \wedge B$. We note $N s b(F)$ this lattice. If $f: F \longrightarrow G$ is a morphism in $\operatorname{Diag}(\mathbf{A b})$, we can define a Galois connection $\left(f_{*}, f^{*}\right)$ where :

- $f_{*}: N s b(F) \longrightarrow N s b(G)$ with $f_{*}(m)=i m(f \circ m)=\operatorname{kercok}(f \circ m)$

- $f_{*}: N \operatorname{sb}(G) \longrightarrow N \operatorname{sb}(F)$ with $f^{*}(n)=\operatorname{ker}((\operatorname{cokn}) \circ f)$

The condition of modularity can be expressed as every morphism $f: F \longrightarrow G$ satisfies :

1) for every $x \in N \operatorname{sb}(F), f^{*} \circ f_{*}(x)=x \vee f^{*}(\perp)$

2) for every $y \in N \operatorname{sb}(G), f_{*} \circ f^{*}(y)=y \wedge f_{*}(\top)$

Theorem 4.20 In $\operatorname{Diag}(\boldsymbol{A b}), 1)$ and 2) fail and so $\operatorname{Diag}(\boldsymbol{A b})$ is not modular.

Proof.

1) Let:

- $F$ the functor from the discrete category $\mathbf{2}$ with two objects to $\mathbf{A b}$ which sends each object to $\mathbb{Z}$

- $G$ the functor from the discrete category $\mathbf{1}$ with one object to $\mathbf{A b}$ which sends this object to $\mathbb{Z}$

- $f: F \longrightarrow G$ the morphism $(\Phi, \sigma)$ where $\Phi$ sends each object of 2 to the unique object of $\mathbf{1}$ and $\sigma$ is the natural transformation from $F$ to $G \circ \Phi$ which is only composed of identities

- $x$ the normal subobject of $F$ which is the functor from $\mathbf{2}$ to $\mathbf{A b}$ which sends each object to 0 (identifying the object with the inclusion in $F$, which is formally the normal subobject)

In this case,

$$
x \vee f^{*}(\perp)=x \neq F=f^{*} \circ f_{*}(x)
$$

2) Let:

- $F$ the functor from $\mathbf{1}$ to $\mathbf{A b}$ which this object to $\mathbb{Z}$ 
- $G$ the functor from the category 2' with two objects $\{a, b\}$ and one non-identity morphism which goes from $a$ to $b$ to $\mathbf{A b}$ which sends each object to $\mathbb{Z}$ and the morphism to identity

- $f: F \longrightarrow G$ the morphism $(\Phi, \sigma)$ where $\Phi$ sends the unique object of $\mathbf{1}$ to $a$ and $\sigma$ is the natural transformation from $F$ to $G \circ \Phi$ which is only composed of identities

- $y$ the normal subobject of $G$ which is the functor from 2' to $\mathbf{A b}$ which sends $a$ to $0, b$ to $\mathbb{Z}$ and the morphism to 0

In this case,

$$
y \wedge f_{*}(\top)=y \neq 0_{G}=f_{*} \circ f^{*}(y)
$$

where $0_{G}: \mathbf{2}$ ' $\longrightarrow \mathbf{A b}$ which maps each object to 0 and the morphism to 0 .

\subsubsection{Relative homology and the exactness axiom}

We denote by $P o T o p_{2}$ the category of pairs $(X, A)$ with $X$ a pospace and $A \subseteq X$ considered as a pospace by restricting the order of $X$ (we call them potopological pairs). A morphism of potopological pairs from $(X, A)$ to $(Y, B)$ is a map $f: X \longrightarrow Y$ with $f(A) \subseteq B$.

Let $(X, A)$ be a potopological pair. As the category $\operatorname{Diag}(\mathbf{A b})$ is homological, so is $C_{\bullet}(\operatorname{Diag}(\mathbf{A b}))$, we can define $\vec{C} \bullet(X, A)=\operatorname{cok}(\operatorname{inj}: \vec{C} \bullet(A) \longrightarrow$ $\vec{C} \bullet(X))$. We could also have defined it as the complex obtained by taking $\operatorname{cok}\left(i n j: \vec{C}_{n}(A) \longrightarrow \vec{C}_{n}(X)\right)$ in $\operatorname{Diag}(\mathbf{A b})$ in each dimension. We have naturally a sequence :

$$
\vec{C} \bullet(A) \stackrel{i n j}{\longrightarrow} \vec{C} \bullet(X) \stackrel{\operatorname{Cok}(i n j)}{\longrightarrow} \vec{C} \bullet(X, A)
$$

which is exact but not short exact. However, if we define

$$
\vec{C}_{\bullet}^{X}(A)=\operatorname{ker}(\operatorname{Cok}(i n j))
$$

we have that $\operatorname{cok}\left(\operatorname{Ker}(\operatorname{Cok}(i n j)): \vec{C}_{\bullet}^{X}(A) \longrightarrow \vec{C} \bullet(X)\right)=\vec{C} \bullet(X, A)$ because $K e r$ and $C o k$ are inverse on normal sub-objects and normal quotients and the sequence :

$$
\vec{C}_{\bullet}^{X}(A) \stackrel{\operatorname{Ker}(\operatorname{Cok}(i n j))}{\longrightarrow} \vec{C} \bullet(X) \stackrel{\operatorname{Cok}(i n j)}{\longrightarrow} \vec{C} \bullet(X, A)
$$

is short exact in $\operatorname{Diag}(\mathbf{A b})$ and so in $\operatorname{Cat}\left(\mathcal{T}_{X}, \mathbf{A b}\right)$, which is abelian. We thus have a long exact sequence : 


$$
\ldots \rightarrow H_{n}(\vec{C} \bullet(X)) \rightarrow H_{n}(\vec{C} \bullet(X, A)) \rightarrow H_{n-1}\left(\vec{C}_{\bullet}^{X}(A)\right) \rightarrow H_{n-1}(\vec{C} \bullet(X)) \rightarrow \cdots
$$

in $\operatorname{Cat}\left(\mathcal{T}_{X}, \mathbf{A b}\right)$ and so in $\operatorname{Diag}(\mathbf{A b})$.

If $f:(X, A) \longrightarrow(Y, B)$ is a morphism of potopological pairs, we can define $f_{\bullet}: \vec{C}_{\bullet}(X, A) \longrightarrow \vec{C}_{\bullet}(Y, B)$ (resp. $f_{\bullet}: \vec{C}_{\bullet}^{X}(A) \longrightarrow \vec{C}_{\bullet}^{Y}(B)$ ) as the unique morphism which makes commutative the right square of the first diagram (resp. the left square of the second) :

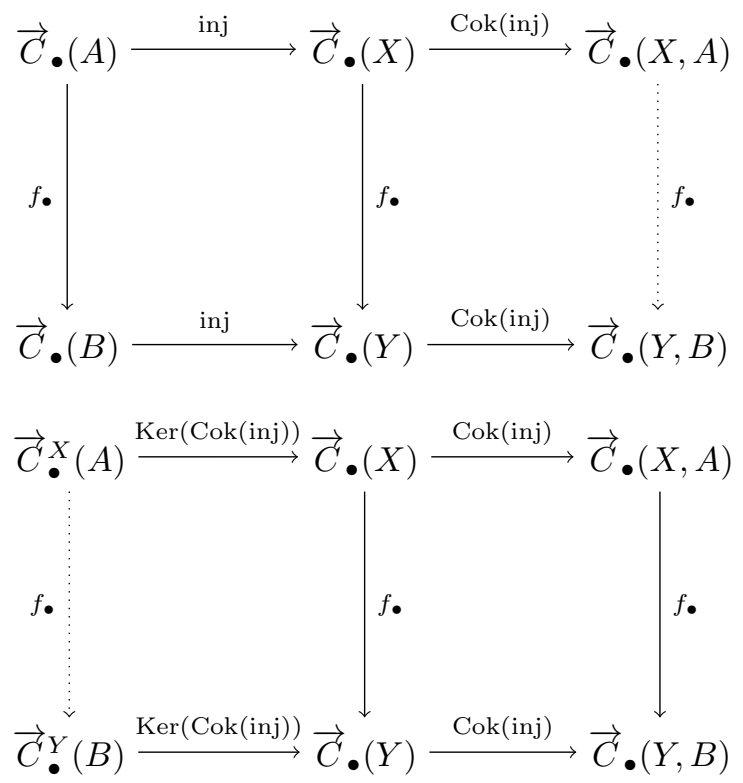

coming from the universal property of $\vec{C} \bullet(X, A)$ (resp. $\vec{C}_{\bullet}^{Y}(B)$ ). This defines two functors from $\mathrm{PoTop}_{2}$ to $\operatorname{Diag}(\mathbf{A b})$. From this, and from Theorem 4.19, we deduce that the long exact sequence of relative homology is natural in $(X, A)$.

\section{Conclusion and future work}

We defined in this paper a notion of directed homology which enjoys many good properties : there are natural homology theories for a large class of pre-cubical sets and for a large class of directed spaces, which agree through geometric realization "modulo bisimulation", and natural homology distinguishes between non-equivalent directed spaces which would not be distinguished using ordinary homology theories (see also [9]). Also, the natural homology of a subdivided pre-cubical set is bisimilar to the natural homology of the original one. We do have Hurewicz theorems linking directed homotopy with natural homology closely,and natural homology enjoys most of Eilenberg-Steenrod axioms for homology theories : dimension, homotopy, additivity and exactness axioms in 
particular. Generally speaking, natural homology maps short exact sequences into some sequences. Unfortunately, they are only order 2 sequences and not long exact sequences, which make calculations slightly more complicated than with ordinary topology. There is nevertheless the hope that some variations of natural homology may agree with modularity, which implies that short exact sequences are mapped onto long exact sequences, for instance some form of natural homology modulo bisimulation may be a good basis for that. This is left for future work, as are some form of the excision axiom, the potentially interesting relationships with persistence homology [6] and the Baues-Wirshing homology theories defined in [27] for studying term rewriting systems, and formalism for describing computations which has some links with the concurrency models which were originally at the basis of this work.

\section{References}

[1] Hans-Joachim Baues and Gunther Wirsching, Cohomology of small categories, Journal of Pure and Applied Algebra, 38(2-3), 187-211 (1985)

[2] Marek A. Bednarczyk, Andrzej M. Borzyszkowski and Wieslaw Pawlowski, Generalized congruences-epimorphisms in Cat, Theory and Applications of Categories 5(11) 266-280 (1999)

[3] Richard Bonichon, Géraud Canet, Loïc Correnson, Eric Goubault, Emmanuel Haucourt, Michel Hirschowitz, Sébastien Labbé and Samuel Mimram, Rigorous Evidence of Freedom from Concurrency Faults in Industrial Control Software, SAFECOMP, 85-98 (2011)

[4] Francis Borceux, Handbook of Categorical Algebra 2 : Categories and Structures. Cambridge University Press (1994)

[5] Glen Bredon, Topology and Geometry. Graduate Texts in Mathematics 139. New York: Springer-Verlag (1993)

[6] Gunnar Carlsson and Afra Zomorodian, Computing Persistent Homology. Discrete and Computational Geometry, 33(2), 249-274 (2005)

[7] E. G. Coffman and Elphick, M. J. and Shoshani, A., System Deadlocks, ACM Computing Surveys 3(2) (1971)

[8] Edsger W. Dijkstra, Cooperating Sequential Processes, Technical Report EWD-123, Technological University Eindhoven (1965) 
[9] Jérémy Dubut and Eric Goubault and Jean Goubault-Larrecq, Natural Homology, ICALP (2) 171-183 (2015)

[10] Ulrich Fahrenberg, Directed Homology. Electronic Notes in Theoretical Computer Science, 100 , 111-125 (2004)

[11] Lisbeth Fajstrup, Dipaths and dihomotopies in a cubical complex, Advances in Applied Mathematics, 35(2), 188-206 (2005)

[12] Lisbeth Fajstrup and Martin Raussen and Eric Goubault, Algebraic topology and concurrency, Theor. Comput. Sci. 357(1-3): 241-278 (2006)

[13] Lisbeth Fajstrup, Eric Goubault, Emmanuel Haucourt, Samuel Mimram, and Martin Raussen, Trace Spaces : An Efficient New Technique for StateSpace Reduction, ESOP, 274-294 (2012)

[14] Lisbeth Fajstrup and Eric Goubault and Emmanuel Haucourt and Samuel Mimram and Martin Raussen, Directed Algebraic Topology and Concurrency, Springer-Verlag (2016)

[15] Lisbeth Fajstrup, Eric Goubault and Martin Raussen, Detecting Deadlocks in Concurrent Systems, CONCUR, 332-347 (1998)

[16] Marco Grandis, General homological algebra, I. Semiexact and homological categories. preprint 186, Dipartimento di Matematica Università degli Studi di Genova (1991)

[17] Marco Grandis, General homological algebra, II. Homology and satellites. preprint 187, Dipartimento di Matematica Università degli Studi di Genova (1991)

[18] Marco Grandis, Directed homotopy theory, I. The fundamental category, Cahiers de Topologie et Géométrie Différentielle Catégoriques, 44, 281-316 (2003)

[19] Marco Grandis, Inequilogical spaces, directed homology and noncommutative geometry. Homology, Homotopy and Applications, 6, 413-437 (2004)

[20] Marco Grandis, Directed Algebraic Topology, Models of non-reversible worlds. Cambridge University Press (2009)

[21] Eric Goubault and Thomas P. Jensen, Homology of Higher Dimensional Automata; CONCUR, 254-268 (1992)

[22] Eric Goubault, Géométrie du parallélisme, PhD thesis, Ecole Polytechnique (1995)

[23] Eric Goubault, Some geometric perspectives in concurrency theory, Homology, Homotopy and Applications, 5, 95-136 (2003). 
[24] Eric Goubault and Emmanuel Haucourt, A Practical Application of Geometric Semantics to Static Analysis of Concurrent Programs, CONCUR, 503-517 (2005)

[25] Eric Goubault and Emmanuel Haucourt, Components of the Fundamental Category II. Applied Categorical Structures, 15(4), 387-414 (2007)

[26] Eric Goubault and Martin Raussen, Dihomotopy as a Tool in State Space Analysis, LATIN, 16-37 (2002)

[27] Yves Guiraud, Philippe Malbos and Samuel Mimram, A Homotopical Completion Procedure with Applications to Coherence of Monoids, RTA, 223-238 (2013)

[28] Maurice Herlihy, Dmitry Kozlov and Sergio Rajsbaum, Distributed Computing Through Combinatorial Topology. Elsevier Science (2013)

[29] André Joyal, Mogens Nielsen and Glynn Winskel, Bisimulation from open maps, Information and Computation, 127(2), 164-185 (1996)

[30] Tomasz Kaczynski, Konstantin Mischaikow and Marian Mrozek, Computing homology, Homology, Homotopy and Applications, 5(2), 233-256 (2003)

[31] Thomas Kahl, The homology graph of a higher dimensional automaton. arXiv :1307.7994 (2013)

[32] S. Krishnan, A convenient category of locally preordered spaces, Applied Categorical Structures, vol. 17 (5), pp 445-466.

[33] Sanjeevi Krishnan, Flow-cut Dualities for Sheaves on Graphs, arXiv:1409.6712 (2014)

[34] S. MacLane, Categories for the working mathematician, Graduate Text in Mathematics, Springer-Verlag, vol. 5, 1971.

[35] Philippe Malbos, Rewriting Systems and Hochschild-Mitchell Homology, Electronic Notes in Theoretical Computer Science, 81, 59-72 (2003)

[36] Fotini Markopoulou, The Internal Description of a Causal Set : What the Universe Looks Like from the Inside, Communications in Mathematical Physics, 211, 559-583 (2000)

[37] Robin Milner, Communication and Concurrency. Prentice Hall, 1989.

[38] Barry Mitchell, Theory of Categories, Academic Press Inc, 1995.

[39] Barry Mitchell, Rings with several objects, Advances in Mathematics, 1972.

[40] Leopoldo Nachbin, Topology and order, volume 4 of Van Nostrand mathematical studies. Robert E. Krieger Publishing Company (1965) 
[41] David Park, Concurrency and Automata on Infinite Sequences, In Deussen, Peter. Theoretical Computer Science. Proceedings of the 5th GI-Conference. Lecture Notes in Computer Science 104, Springer-Verlag, 1981.

[42] Vaughan R. Pratt, Modeling Concurrency with Geometry, POPL, 311-322 (1991)

[43] Martin Raussen, Trace spaces in a pre-cubical complex. Topology and its Applications, 156(9), 1718-1728 (2009)

[44] Martin Raussen, Simplicial models for trace spaces. Algebraic and Geometric Topology, 10(3), 1683-1714 (2010)

[45] Martin Raussen, Simplicial models for trace spaces II : General higher dimensional automata. Algebraic and Geometric Topology, 12(3), 1741-1762 (2012)

[46] Christine Tasson, Wait-free protocols : from discrete to topological semantics. technical report, CEA Saclay (2010)

[47] Rob J. van Glabbeek, On the Expressiveness of Higher Dimensional Automata : (Extended Abstract). Electronic Notes in Theoretical Computer Science, 128(2), 5-34 (2005)

\section{$.1 \quad$ The category of diagrams}

As well known [34], Cat is complete.

As a matter of fact, if $\mathcal{C}$ is a small category and $F: \mathcal{C} \longrightarrow$ Cat a diagram in Cat, the limit of $F$ is the category $\mathcal{L}_{F}$ whose :

- objects are the families $\left(x_{c}\right)_{c \in O b(\mathcal{C})}$ where $x_{c} \in O b(F(c))$ and for every morphism $f: c \longrightarrow c^{\prime}$ of $\mathcal{C}, F(f)\left(x_{c}\right)=x_{c^{\prime}}$

- morphisms from $\left(x_{c}\right)_{c \in O b(\mathcal{C})}$ to $\left(y_{c}\right)_{c \in O b(\mathcal{C})}$ are the families $\left(g_{c}\right)_{c \in O b(\mathcal{C})}$ where $g_{c}: x_{c} \longrightarrow y_{c}$ and for every morphism $f: c \longrightarrow c^{\prime}$ of $\mathcal{C}, F(f)\left(g_{c}\right)=$ $g_{c^{\prime}}$

- composition $\left(h_{c}\right)_{c \in O b(\mathcal{C})} \circ\left(g_{c}\right)_{c \in O b(\mathcal{C})}$ is $\left(h_{c} \circ g_{c}\right)_{c \in O b(\mathcal{C})}$

- identity of $\left(x_{c}\right)_{c \in O b(\mathcal{C})}$ is $\left(i d_{x_{c}}\right)_{c \in O b(\mathcal{C})}$

together with the projection maps.

We are now ready to define limits in $\operatorname{Diag}(\mathcal{M})$.

Definition $.1(\mathcal{C}$-diagram, $\mathcal{C}$-limit) $A \mathcal{C}$-diagram of $\mathcal{M}$ is a functor from $\mathcal{C}$ to $\mathcal{M}$. We say that a category $\mathcal{M}$ has $\mathcal{C}$-limits if every $\mathcal{C}$-diagram of $\mathcal{M}$ has a limit.

Now, $\mathcal{C}$-limits of diagrams in $\mathcal{M}$ are fully determined by $\mathcal{C}$-limits in $\mathcal{M}$ : 
Theorem .2 (Preservation of limits) Let $\mathcal{C}$ be a small category. $\operatorname{Diag}(\mathcal{M})$ has $\mathcal{C}$-limits iff $\mathcal{M}$ has $\mathcal{C}$-limits.

Proof. First, suppose that $\operatorname{Diag}(\mathcal{M})$ has $\mathcal{C}$-limits. Let $F: \mathcal{C} \longrightarrow \mathcal{M}$. We consider $G: \mathcal{C} \longrightarrow \operatorname{Diag}(\mathcal{M})$ the following $\mathcal{C}$-diagram:

- for every object $c$ of $\mathcal{C}, G(c)$ is the diagram from the category $\mathbf{1}$ with one object and one morphism to $\mathcal{M}$ which maps this object to $F(c)$

- for every morphism $f: c \longrightarrow c^{\prime}$ of $\mathcal{C}, G(f)$ is the morphism

$$
\left(i d_{\mathbf{1}},(F(f))_{* \in O b(\mathbf{1})}\right)
$$

As $\operatorname{Diag}(\mathcal{M})$ has $\mathcal{C}$-limits, $G$ has a limit $\left(L, \Pi_{c}: L \longrightarrow G(c)\right)$. Necessarily, $L$ is from 1 to $\mathcal{M}$ and so is just an object $K$ of $\mathcal{M}$ and the $\Pi_{c}$ are just morphism $\pi_{c}: K \longrightarrow F(c)$. It is easy to see that $\left(K, \pi_{c}\right)$ is the limit of $F$.

Now, suppose that $\mathcal{M}$ has $\mathcal{C}$-limits. Let $F: \mathcal{C} \longrightarrow \operatorname{Diag}(\mathcal{M}) . F$ induces a $\mathcal{C}$-diagram in Cat $G_{F}: \mathcal{C} \longrightarrow$ Cat in the following way:

- $G_{F}(c)$ is the domain of $F(c)$

- $G_{F}(f)$ is the first component of $F(f)$

As Cat is complete, $G_{F}$ has a limit $\left(\mathcal{L}_{G_{F}}, \pi_{c}: \mathcal{L}_{G_{F}} \longrightarrow G(c)\right)$. Now, the limit of $F$ is the functor $\mathcal{L}_{F}: \mathcal{L}_{G_{F}} \longrightarrow \mathcal{M}$ such that:

- $\mathcal{L}_{F}\left(\left(x_{c}\right)_{c \in O b(\mathcal{C})}\right)$ is the limit of the functor which maps

* every $c \in O b(\mathcal{C})$ to $F(c)\left(x_{c}\right)$

* every $f: c \longrightarrow c^{\prime}$ of $\mathcal{C}$ to the component of $x_{c}$ in the second component of $F(f)$ (which is a natural transformation).

Such a limit exists because $\mathcal{M}$ has $\mathcal{C}$-limits.

- $\mathcal{L}_{F}\left(\left(g_{c}\right)_{c \in O b(\mathcal{C})}\right)$ (where $g_{c}: x_{c} \longrightarrow y_{c}$ ) is the unique morphism of $\mathcal{M}$ defined below. As $\mathcal{L}_{F}\left(\left(y_{c}\right)_{c \in O b(\mathcal{C})}\right)$ is a limit in $\mathcal{M}$, it makes every such diagram:

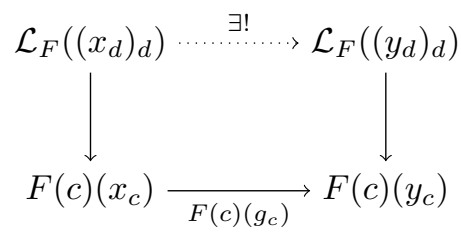

commutative, together with the projection maps

$$
\left(\pi_{c},\left(\sigma_{c,\left(x_{d}\right)_{d}}\right)_{\left(x_{d}\right)_{d} \in O b\left(\mathcal{L}_{G_{F}}\right)}\right): \mathcal{L}_{F} \longrightarrow F(c)
$$

where $\sigma_{c,\left(x_{d}\right)_{d}}: \mathcal{L}_{F}\left(\left(x_{d}\right)_{d}\right) \longrightarrow F(c)\left(x_{c}\right)$ is the projection map coming from the fact that $\mathcal{L}_{F}\left(\left(x_{d}\right)_{d}\right)$ is a limit. 
Therefore, a direct consequence is that $\operatorname{Diag}(\mathcal{M})$ is complete iff $\mathcal{M}$ is complete.

Similarly, we know that Cat is cocomplete [34].

We briefly recap the construction below. We need first to define congruences on categories.

If $f: c \longrightarrow c^{\prime}$, we note $\operatorname{dom}(f)=c$ and $\operatorname{cod}(f)=c^{\prime}$. If $\sim$ is a equivalence relation on the set $O b(\mathcal{C})$ of a small category $\mathcal{C}$, we note $\operatorname{Mor}_{+}(\mathcal{C})$, the set of non-empty finite sequences of morphisms of $\mathcal{C}\left(f_{1}, \ldots, f_{n}\right)$.

A congruence on the small category $\mathcal{C}$ is a pair $\left(\sim_{o}, \sim_{m}\right)$ of an equivalence relation $\sim_{0}$ on $\mathrm{Ob}(\mathcal{C})$ and a partial equivalence relation $\sim_{m}$ on $\mathrm{Mor}_{+}(\mathcal{C})$ satisfying:

- if $\left(f_{0}, \ldots, f_{n}\right) \sim_{m}\left(g_{1}, \ldots, g_{p}\right)$ then for all $i \leq n, \operatorname{dom}\left(f_{i}\right) \sim_{o} \operatorname{dom}\left(f_{i-1}\right)$

- $\left(f_{0}, \ldots, f_{n}\right) \sim_{m}\left(g_{0}, \ldots, g_{p}\right)$ implies $\operatorname{dom}\left(f_{0}\right) \sim_{o} \operatorname{dom}\left(g_{0}\right)$ and $\operatorname{cod}\left(f_{n}\right) \sim_{o}$ $\operatorname{cod}\left(g_{p}\right)$

- $c \sim_{0} c^{\prime}$ implies $i d_{c} \sim_{m} i d_{c^{\prime}}$

- $\left(f_{0}, \ldots, f_{n}\right) \sim_{m}\left(g_{0}, \ldots, g_{p}\right),\left(f_{n+1}, \ldots, f_{n^{\prime}}\right) \sim_{m}\left(g_{p+1}, \ldots, g_{p^{\prime}}\right)$ and $\operatorname{cod}\left(f_{n}\right) \sim_{o}$ $\operatorname{dom}\left(f_{n+1}\right)$ then $\left(f_{0}, \ldots, f_{n^{\prime}}\right) \sim_{m}\left(g_{0}, \ldots, g_{p^{\prime}}\right)$

- if $\operatorname{cod}(f)=\operatorname{dom}(g)$ then $(f, g) \sim_{m}(g \circ f)$

Proposition $.3([2])$ If $\left(\sim_{0}, \sim_{m}\right)$ is a congruence on $\mathcal{C}$, the category $\mathcal{C} /\left(\sim_{o}\right.$ ,$\left.\sim_{m}\right)$ whose:

- objects are equivalence class of objects of $\mathcal{C}$ modulo $\sim_{o}$, written [-]

- morphisms from $[c]$ to $\left[c^{\prime}\right]$ are equivalence class of elements $\left(f_{1}, \ldots, f_{n}\right)$ of $\operatorname{Mor}_{+}(\mathcal{C})$ such that $\operatorname{dom}\left(f_{1}\right) \sim_{o} c$ and $\operatorname{cod}\left(f_{n}\right) \sim_{0} c^{\prime}$, written $\left[f_{1}, \ldots, f_{n}\right]$

- composition $\left[g_{1}, \ldots, g_{p}\right] \circ\left[f_{1}, \ldots, f_{n}\right]$ is $\left[f_{1}, \ldots, f_{n}, g_{1}, \ldots, g_{p}\right]$

- identity on $[c]$ is $\left[i d_{c}\right]$

is well defined and is called quotient of $\mathcal{C}$ by $\left(\sim_{o}, \sim_{m}\right)$

Proof. [of Proposition .1] First, it is known [2] that if $R_{o}$ is a relation on $O b(\mathcal{C})$ and $R_{m}$ is a relation on $\mathrm{Mor}_{+}(\mathcal{C})$ then there exists a least congruence on $\mathcal{C}$ that contains $\left(R_{o}, R_{m}\right)$.

Now, let $\mathcal{C}$ be a small category and $F: \mathcal{C} \longrightarrow$ Cat a diagram in Cat. The colimit $\Gamma_{F}$ of $F$ is the quotient of the disjoint union of all the $F(c)$ by the least congruence $\left(\sim_{o}, \sim_{m}\right)$ such that:

- if $f: c \longrightarrow c^{\prime}$ is a morphism of $\mathcal{C}$, then for every object $x_{c}$ of $F(c)$, $F(f)\left(x_{c}\right) \sim_{o} x_{c}$

- if $f: c \longrightarrow c^{\prime}$ is a morphism of $\mathcal{C}$, then for every morphism $g_{c}$ of $F(c)$, $\left(F(f)\left(g_{c}\right)\right) \sim_{m}\left(g_{c}\right)$ 
As in limits, a colimit of a diagram in $\operatorname{Diag}(M)$ is defined as a functor from the colimit of the diagram induced in Cat. Then the functor maps each object of this colimit to the colimit in $\mathcal{M}$ of a diagram which depends of this object and is much more complicated than the initial diagram. Then, derived similarly as in the case of limits :

Theorem .4 If $\mathcal{M}$ is cocomplete then Diag $(M)$ is cocomplete. 